OPEN ACCESS

Edited by:

Andrea Di Nisio,

University of Padua, Italy

Reviewed by:

Alberto Mantovani,

National Institute of Health (ISS), Italy

Cristina Canova,

University Padova, Italy

*Correspondence:

Young Ah Lee

nina337@snu.ac.kr

Specialty section:

This article was submitted to

Translational Endocrinology,

a section of the journal

Frontiers in Endocrinology

Received: 20 March 2021

Accepted: 16 August 2021

Published: 09 September 2021

Citation:

Lee YJ, Jung HW, Kim HY, Choi Y-J and Lee YA (2021) Early - Life Exposure to Per- and PolyFluorinated Alkyl Substances and Growth, Adiposity, and Puberty in Children: A Systematic Review.

Front. Endocrinol. 12:683297. doi: 10.3389/fendo.2021.683297

\section{Early-Life Exposure to Per- and Poly- Fluorinated Alkyl Substances and Growth, Adiposity, and Puberty in Children: A Systematic Review}

\author{
Yun Jeong Lee ${ }^{1}$, Hae Woon Jung ${ }^{2}$, Hwa Young Kim ${ }^{1}$, Yoon-Jung Choi ${ }^{3,4}$ \\ and Young Ah Lee ${ }^{1 *}$ \\ ${ }^{1}$ Department of Pediatrics, Seoul National University Children's Hospital, Seoul National University College of Medicine, \\ Seoul, South Korea, ${ }^{2}$ Department of Pediatrics, Kyung Hee University Medical Center, Seoul, South Korea, ${ }^{3}$ Department of \\ Preventive Medicine, Seoul National University College of Medicine, Seoul, South Korea, ${ }^{4}$ Environmental Health Center, \\ Seoul National University College of Medicine, Seoul, South Korea
}

Per- or polyfluoroalkyl substances (PFAS), a family of synthetic polyfluorinated compounds, are widely used in consumer products. Ubiquitous exposures to PFAS, in consideration of their persistence, bioaccumulation potential, and toxicities have led to concerns regarding possible harmful effects during critical periods of development in early-life and long-term consequences on health. The potential effects of PFAS depend on various factors including the type of PFAS and the timing and level of exposure. We performed a systematic review of the epidemiologic literature to assess the effects of earlylife PFAS exposure on prenatal and postnatal growth, adiposity, and puberty in children and adolescents. For birth size, most studies indicated that prenatal PFAS exposure, in particular long-chain PFAS, may impair fetal growth, albeit some reports of null associations with maternal PFAS. For growth within 2 years of age, prenatal PFAS exposure showed no associations with height and either null or negative associations with weight. However, postnatal PFAS exposures were inversely related to height and weight at 2 years in a cross-sectional study. For postnatal adiposity, prenatal PFAS may mostly have negative associations with body mass index in the first 2 years of life, but positive relationships with adiposity in childhood and adolescence, although some studies showed null associations. For puberty, the evidence for associations between early-life PFAS exposure and pubertal development or sex hormone levels were limited and inconclusive. From experimental studies, plausible mechanisms through which PFAS may affect early-life growth and puberty include PFAS-induced activation of peroxisome proliferator-activated receptor, alterations of thyroid or steroid hormone synthesis and metabolism, and their weak estrogenic or anti-androgenic properties. Although the published literature suggests possible effects of PFAS exposures on early-life growth, adiposity, and puberty, current human evidence is limited in establishing PFAS-induced effects on early-life physical development. Further investigation is warranted to clarify PFAS-induced effects on growth and physical development in consideration of the critical 
time-window of exposure, concomitant exposure to chemical mixtures including various PFAS types, and possible non-monotonic dose-response relationship for growth and adiposity trajectories.

Keywords: perfluorinated alkylated substances, birth weight, growth, adiposity, puberty, child, adolescent

\section{INTRODUCTION}

Per- or polyfluoroalkyl substances (PFAS) are a group of highly stable synthetic polyfluorinated compounds that exhibit unique physical and chemical characteristics, including water and oil repellency, thermal stability and surfactant properties (1). Since their first production in the 1940s and 1950s, PFAS have been incorporated into numerous products such as food packaging material, cookware, clothing, carpets, and fire extinguishers (1). By the early 2000s, PFAS became broadly distributed in the environment (2) and virtually all people living in industrialized countries were exposed to many PFAS, with blood concentrations in the $\mathrm{ng} / \mathrm{ml}$ range (3).

The molecular structure of PFAS consists of a chain of carbon atoms linked to fluorine atoms. By component, PFAS are grouped into perfluoroalkyl carboxylic acids (PFCA) and perfluoroalkyl sulfonic acids (PFSA). The so called "longchain" PFAS (PFCA with $\geq 7$, and PFSA with $\geq 6$ perfluorinated carbons) which includes perfluorooctanoate (PFOA), perfluorononanoic acid (PFNA), perfluorodecanoic acid (PFDA), perfluoroundecanoic acid (PFUnDA), perfluorododecanoic acid (PFDoDA), perfluorooctane sulfonic acid (PFOS), perfluoroheptanane sulfonic acid (PFHpS), and perfluorohexane sulfonic acid (PFHxS), have been reported have more bioaccumulation potential and toxicities than "shortchain" PFAS (4). Although serum levels of PFOA and PFOS, the most widely used long-chain PFAS, are declining in some countries since the early 2000s $(5,6)$, the use of short-chain PFAS or other novel PFAS have been increasing (7).

PFAS are resistant to environmental degradation and remain in the human body for a long time. Their half-lives are 3.8, 5.4, and 8.5 years for PFOA, PFOS, and PFHxS, respectively (8). Human exposure to PFAS may occur via drinking water, diet, indoor and outdoor air, house dust, and consumer products (9), and maternal PFAS can be transferred to the fetus across the placental barrier (10). Young children are likely to have higher PFAS body burdens than adults due to cumulative exposure via placental transfer and breastfeeding, higher water intake relative to body size, and more inhalation or ingestion of house dust due to their behavior (11).

Ubiquitous exposures to PFAS and their bioaccumulation potential have led to concerns regarding potential toxicities and health effects, particularly in growing children (12). In line with the "Developmental origins of health and diseases (DOHaD)" hypothesis (13), early-life growth and development may be more vulnerable to exposure to PFAS, with subsequent adverse health outcomes in later adulthood. We review the current epidemiologic evidence of the effects of prenatal and postnatal PFAS exposures on growth, adiposity, and puberty in children.

\section{METHODS}

This systematic review was reported according to Preferred Reporting Items for Systematic Review and meta-Analysis (PRISMA) guidelines (14).

\section{Definition}

Exposure was defined as exposure to PFOA, PFNA, PFDA, PFUnDA, PFDoDA, perfluorotridecanoic acid (PFTrDA), perfluorotetradecanoic acid (PFTeDA), perfluorohexadecanoic acid (PFHxDA), PFOS, PFHpS, PFHxS, perfluorodecane sulfonic acid (PFDS), pentafluorobenzoic acid (PFBA), perfluoropentanoic acid (PFPeA), perfluorohexanoic acid (PFHxA), perfluoroheptanoic acid (PFHpA), perfluorobutane sulfonic acid (PFBS), perfluorooctane sulfonamide (PFOSA), $\mathrm{N}$-ethyl-perfluorooctane sulfonamidoacetic acid (Et-PFOSA$\mathrm{AcOH}$ ), or N-methyl-perfluorooctane sulfonamidoacetic acid (Me-PFOSA-AcOH). For outcome measures, fetal growth was evaluated by measures of birth weight, birth length, ponderal index, and gestational age; postnatal growth by measures of height/length and weight; adiposity by measures of body mass index (BMI), ponderal index, waist circumference, skinfold thickness, and fat mass; pubertal outcomes by measures of pubertal stage, age at menarche, and levels of sex-hormones.

\section{Data Sources and Selection}

We searched MEDLINE, EMBASE, Web of Science, and Scopus on May 21, 2021 with the keywords shown in Supplementary Table 1. The selection criteria were 1) cohort studies or casecontrol studies which investigated the effects of PFAS on growth, adiposity, or puberty in children and adolescents, 2) primary research articles in English and published in peer-reviewed journals. Two authors independently screened titles and abstracts using EndNote and reviewed full-text articles. Disagreements were resolved through discussion.

\section{Data Extraction and Risk of Bias Assessment}

Two reviewers independently extracted the data including country, sample size, study design, timing of exposure, exposure measures, the level of exposure of PFAS, and outcome measures. Outcomes were expressed in terms of the odds ratio, relative risk, or $\beta$, and described in the summary of findings. We assessed the quality of the studies using the Cochrane Risk of Bias In Non-randomized Studies-of Exposure (ROBINS-E) tool (15). The preliminary template for ROBINS-E is available online (16). Disagreements in the assessment by the two authors were resolved through discussion and opinions of a third investigator were considered if needed. For risk of bias 
assessment, each domain of risk of bias was determined to be low, moderate, or high. Overall risk of bias was considered high if there was high risk in any of the seven domains, and low if there was low risk of bias in all seven domains.

\section{RESULTS}

\section{Study Selection}

A total of 1534 articles were identified in the database. After excluding duplications $(\mathrm{n}=993), 542$ records were screened (Supplementary Figure 1). These articles were screened by title ( 376 were excluded), abstract ( 73 were excluded) and full text (3 were excluded). The reasons for exclusion are shown in Supplementary Figure 1. The 90 studies included in this review were published between November 2007 and July 2021.

\section{Characteristics of Included Studies}

The 90 studies were comprised of 62 cohort studies, 22 crosssectional studies, and 6 case-control studies. Sample sizes ranged from 70 to 6007. Twenty-two studies were performed in the United States, 12 in Denmark, and 8 in China. To assess for the effects of early life exposure to PFAS, 61 studies used maternal blood samples, 16 studies used cord blood samples, 11 studies used children's blood samples, and 1 study used both maternal and childhood samples. In terms of outcomes, 49 studies evaluated for fetal growth, 5 studies for postnatal growth, 19 studies for postnatal adiposity, 6 studies for both fetal and postnatal growth or adiposity, and 11 studies for pubertal outcomes or sex-hormone levels. Among various PFAS substances, the effects of PFOA and PFOS were most frequently evaluated, followed by PFHxS and PFNA. The median exposure levels of PFOA, PFOS, PFHxS, and PFNA in maternal blood samples were ranged from $0.2-21.1 \mathrm{ng} / \mathrm{mL}, 0.8-33.4 \mathrm{ng} / \mathrm{mL}, 0.1$ $4.5 \mathrm{ng} / \mathrm{mL}$, and 0.1-2.3 ng/mL, respectively. In childhood samples, the median exposure levels were $0.5-34.8 \mathrm{ng} / \mathrm{mL}$ for PFOA, 0.8-44.5 $\mathrm{ng} / \mathrm{mL}$ for PFOS, $0.2-8.1 \mathrm{ng} / \mathrm{mL}$ for PFHxS, and $0.3-1.3 \mathrm{ng} / \mathrm{mL}$ for PFNA, respectively. Exposure levels for various PFAS substances of each study are described in Supplementary Materials (Supplementary Tables 2-5). The details of included articles and the results of risk of bias assessment are also shown in Supplementary materials (Supplementary Tables 2-9).

\section{Fetal Growth}

The effects of prenatal PFAS exposures on fetal growth have been much studied over the past few decades. We included 52 studies for a review of birth outcomes. Prenatal exposure to PFAS was assessed during gestation from maternal blood (first to third trimester) or cord blood samples (Table 1 and Supplementary Table 2). Birth outcome measures included birth weight, birth length, ponderal index, gestational age, low birth weight, small for gestational age, or preterm birth.

Studies that analyzed the relationship between PFAS exposures and birth weight have mostly shown inverse or null associations, although the results differed by sex, type of PFAS, and timing of exposure. Eleven studies reported inverse associations between PFOA exposure and birth weight (17-20,
$28-32,44,45)$, while other studies showed null associations (21$26,33-43,46-51)$. Two recent meta-analyses of 16 and 24 studies $(69,70)$, found decreases in birth weight of $12.8 \mathrm{~g}(69)$ and $10.5 \mathrm{~g}$ (70) with $1 \mathrm{ng} / \mathrm{ml}$ increases in PFOA in maternal and cord blood, respectively. For PFOS, 14 studies showed inverse relationships with birth weight $(18,20,23,31-33,36,43-46,48-50)$, while 18 showed no associations $(17,19,21,22,24-26,29,30,34,35,37$, 39-42, 47, 51). A meta-analysis of nine studies showed a $0.9 \mathrm{~g}$ decrease in birth weight per $1 \mathrm{ng} / \mathrm{ml}$ increases in maternal PFOS exposures (69).

A few studies included analyses of long-chain PFAS other than PFOA or PFOS, such as PFNA, PFDA, PFUnDA, PFDoDA, PFTrDA, PFHpS, PFHxS, or Me-PFOSA-AcOH. Despite inconsistencies, the relationship between these long-chain PFAS and birth weight was mostly inverse $(18,20,23,32,38$, $40,44)$, a finding which was dominant in girls $(20,32,38,40)$, while other studies showed null associations (19, 21, 22, 24-26, 29, 34, 37, 41-43, 46, 47, 51). Meanwhile, short-chain PFAS such as PFBA, PFHpA, or PFBS were only evaluated in four studies and showed no associations with birth weight $(20,26,29,44)$. Only one study assessed the effects of PFAS as mixtures using data from the Health Outcomes and Measures of Environment (HOME) study (52) and reported a small decrease in birth weight with increased exposure to a mixture of 5 PFAS (PFOA, PFNA, PFDA, PFOS, and PFHxS).

The effects of PFAS exposures on birth length, ponderal index, or gestational age were evaluated. Birth length or the ponderal index showed inverse relationships with PFOA or PFOS exposures (2628, 30, 32, 37, 43, 45, 48) and PFNA, PFDA, PFUnDA, PFDoDA, or PFHxS exposures $(26,32,37,40,47)$, or null associations $(19,22,24$, $38,39,41,46)$, although one study reported a positive relationship between PFHxS exposure and birth length in boys (47). For gestational age, some studies reported increased risk for preterm birth and lower gestational age with increasing exposure to PFOA, PFNA, PFOS or PFHpS in maternal blood $(18,23,28,42,46)$, however, no associations were demonstrated in most studies $(24,31$, 32, 34, 35, 39, 41, 45, 49, 50).

Taken together, despite some inconsistency, a preponderance of studies suggest that maternal PFAS exposure, particularly to long-chain PFAS including PFOA and PFOS, negatively impact fetal growth. However, studies investigating short-chain PFAS are limited, showing no significant effects on fetal growth.

\section{Postnatal Growth During Infancy and Childhood}

We reviewed 12 studies with outcome measures of weight, length and/or height in infancy ( $<2$ years) and childhood (2-12 years). In 10 studies, prenatal PFAS exposure was measured in maternal blood (during gestation or shortly after birth) or in cord blood samples taken at birth the time of birth. Two cross-sectional studies quantified exposure to PFAS in the child's blood $(71,72)$. The most frequently studied PFAS was PFOA, which was quantified in all the studies that were reviewed. Many of the studies also quantified and analyzed other long-chain PFAS, such as PFOS, PFNA, PFDA, and PFHxS with high detection rates. Only a few of the studies included PFUnDA, PFDoDA, Me- 
TABLE 1 | Summary of studies assessing associations between prenatal PFAS exposure and fetal growth.

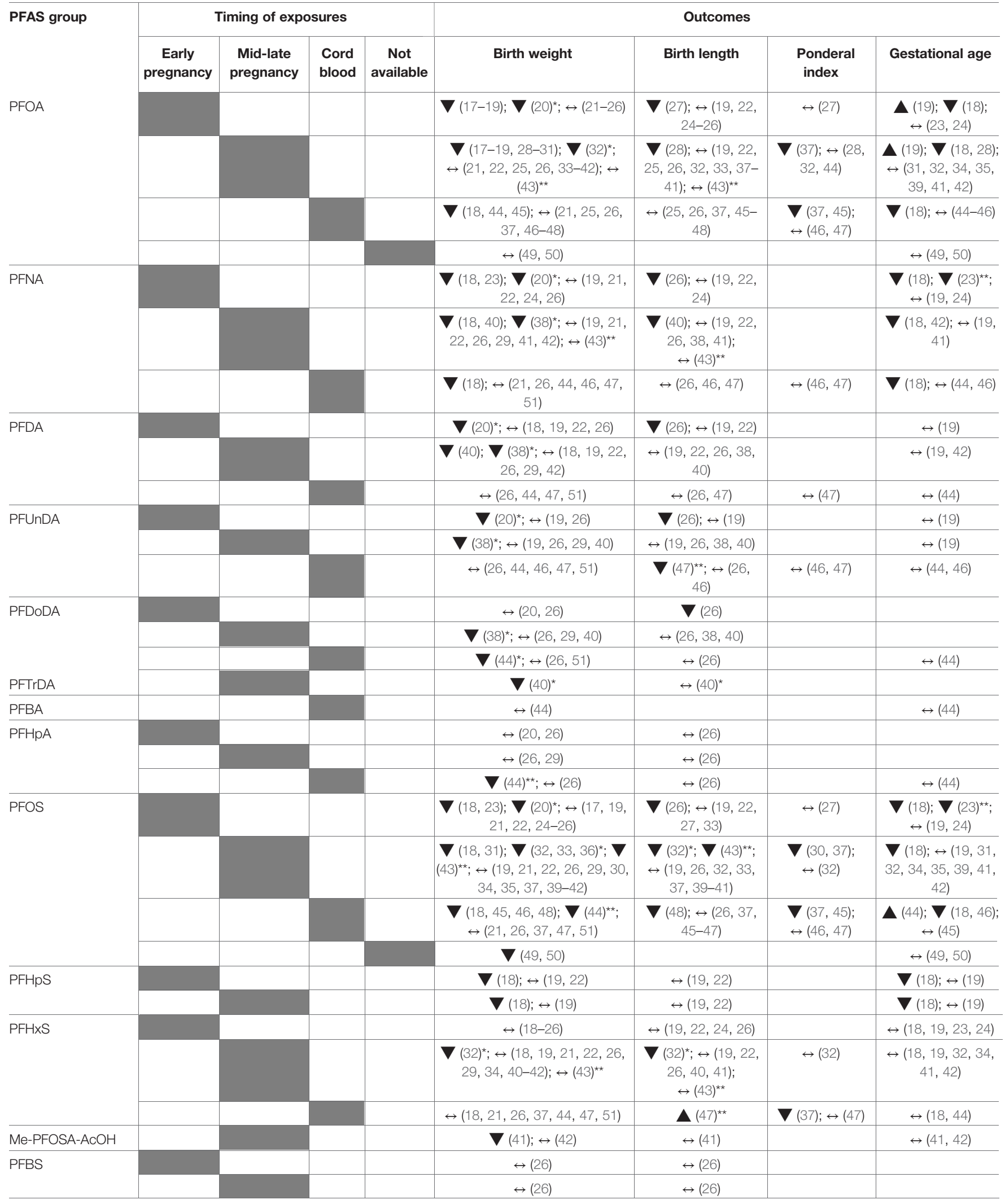


TABLE 1 | Continued

\begin{tabular}{|c|c|c|c|c|c|c|c|c|}
\hline \multirow[t]{3}{*}{ PFAS group } & \multicolumn{4}{|c|}{ Timing of exposures } & \multicolumn{4}{|c|}{ Outcomes } \\
\hline & $\begin{array}{c}\text { Early } \\
\text { pregnancy }\end{array}$ & $\begin{array}{c}\text { Mid-late } \\
\text { pregnancy }\end{array}$ & $\begin{array}{l}\text { Cord } \\
\text { blood }\end{array}$ & $\begin{array}{c}\text { Not } \\
\text { available }\end{array}$ & Birth weight & Birth length & $\begin{array}{l}\text { Ponderal } \\
\text { index }\end{array}$ & Gestational age \\
\hline & & & & & $\leftrightarrow(26)$ & $\leftrightarrow(26)$ & & \\
\hline Mixture & & & & & $\nabla(52)$ & & & \\
\hline
\end{tabular}

PFOA, perfluorooctanoic acid; PFNA, perfluorononanoic acid; PFDA, perfluorodecanoic acid; PFUnDA, perfluoroundecanoic acid; PFDoDA, perfluorododecanoic acid; PFTrDA, perfluorotridecanoic acid; PFBA, pentafluorobenzoic acid; PFHpA, perfluoroheptanoic acid; PFOS, perfluorooctane sulfonic acid; PFHpS, perfluoroheptanane sulfonic acid; PFHxS, perfluorohexane sulfonic acid; Et-PFOSA-AcOH, N-ethyl-perfluorooctane sulfonamidoacetic acid; Me-PFOSA-AcOH, N-methyl-perfluorooctane sulfonamidoacetic acid; PFBS, perfluorobutane sulfonic acid $\mathbf{\Lambda}$, Positive associations; $\mathbf{\nabla}$, Inverse association; $\leftrightarrow$, Null association; * significant only in girls; ** significant only in boys.

Early and mild-late pregnancy refer to $1^{\text {st }}$ trimester, and $2^{\text {nd }}$ or $3^{\text {rd }}$ trimester, respectively.

Long-chain PFAS include PFOA, PFNA, PFDA, PFUnDA, and PFDoDA in perfluoroalkyl carboxylic acids (PFCA) group, and PFOS, PFHpS, PFHxS, Et-PFOSA-AcOH, and Me-PFOSAAcOH in perfluoroalkane sulfonic acids (PFSA) group. Short-chain PFAS include PFBA and PFHpA in PFCA group, and PFBS in PFSA group.

Shading boxes indicate exposure timing.

Four studies with small sample size (less than 100) (53-56), 6 studies investigating the effects of mixture of various chemicals (57-62), and 6 studies focusing on the mediating factors (6368) are not described in the table. Further information of the included studies are described in Supplementary Table 2.

PFOSA-AcOH, PFHpA, PFDS, and PFBS (Table 2 and Supplementary Table 3).

\section{Postnatal Length/Height}

For early postnatal growth, there have been seven studies that analyzed for length/height outcomes by measures of postnatal length/height (in cm) or height z-scores. Six studies assessed for associations between prenatal PFAS exposures and length/height within the first 2 years of life. All of the studies that assessed for gestational PFOA and PFOS exposures by sampling maternal blood or cord blood showed null associations with measures of the child's length after birth to 2 years $(48,73,74,76-78)$. The lack of associations to length measures was also seen in other PFAS including PFNA, PFDA, PFTrDA, PFTeDA, PFHxDA, PFHxS, PFDS, and PFBS in the same studies. The only study that reported any significant associations between prenatal exposure and postnatal length was by Cao et al., in which length at 19 months of age was positively associated with the highest tertiles of cord blood PFDoDA (in boys) and PFUnDA (in girls). However, the results were not consistent, as the same study also noted an inverse association between higher exposure to PFDS and postnatal length (77). Only one cross-sectional study, in which growth parameters was analyzed according to the children's PFAS concentrations, showed consistent doseresponse inverse associations of PFOA, PFNA, PFDA, PFOS, and PFHxS with height at 2 years of age (71).

For childhood growth past 2 years, four studies have been conducted $(38,48,72,76)$ with conflicting results. Two studies continued to assess for the effects of prenatal PFAS exposures on height measures in childhood past 2 years by including participant data up to 5 years (76) and 9 years (48) of age. While the study by Gyllenhammer et al. of the Persistent Organic Pollutants in Uppsala Primiparas (POPUP) cohort continued to show no associations between PFAS (PFOA, PFNA, PFDA, PFUnDA, PFOS, PFHxS, and PFBS) and height up to 5 years of age (76), Chen et al. noted that the height in boys of the Taiwan Birth Pnel Study (TBPS) between 24 to 60 months and between 60 and 108 months were both positively associated with cord blood PFOS (48). In contrast, Wang et al. reported inverse associations of prenatal ( $3^{\text {rd }}$ trimester) exposures to
PFDA, PFUnDA and PFDoDA with height z-scores from 2 to 11 years of age in girls, without significant interactions by age (38). A cross-sectional study using data from the National Health and Nutrition Examination Survey (NHANES, 2013-2014) of the United States also reported inverse associations between PFOS and PFHxS exposures and height $\mathrm{z}$-scores in children 3-11 years old (72).

Most of studies to date with regards to postnatal length/height within the first 2 years of life suggests the absence of significant associations with maternal or cord blood PFAS levels. Only one cross-sectional study demonstrated inverse associations between PFAS exposures and height at 2 years. Few studies analyzed for changes in height into later childhood and they showed conflicting associations with PFAS levels.

\section{Postnatal Weight}

Studies that analyzed postnatal weight have found either null or inverse associations between PFAS and measures of weight (weightfor-age, weight-for-length, and change in weight z-scores) (Table 2 and Supplementary Table 3). For prenatal PFOA exposure, the majority of the studies noted no associations with weight parameters during the first 2 years of life $(32,48,73,75,76,78)$. However, two studies did demonstrate consistently inverse associations between PFOA and weight before 2 years of age. In a study of 334 infants with repeated anthropometric measurements from 4 weeks to 2 years of age, prenatal PFOA exposure was inversely associated with weight-for-age or weight-for-height (74). PFOA was also inversely associated with postnatal weight in boys at $19.7 \pm 3.2$ months of age, however the association was significant only between the second and first tertiles of PFOA (77). For PFOS, there were more studies that suggested associations with weight in the first 2 years $(32,48,73-75)$ as opposed to null associations (7678). Prenatal PFOS exposures were inversely related to weight measures in 5 month-old girls (75), in both sexes between 4 weeks and 2 years (74) and at 12 months (73). Similarly, weight $\mathrm{z}$-scores in girls significantly decreased from 6 to 12 months $(-0.25$, $95 \%$ CI $-0.47,-0.04)$ and 12 to 24 months $(-0.24,95 \%$ CI -0.41 , -0.06) per ln-unit increase in cord blood PFOS (48). One study contrastingly showed a positive association between first trimester 
TABLE 2 | Summary of studies assessing associations between PFAS exposure and postnatal growth outcomes.

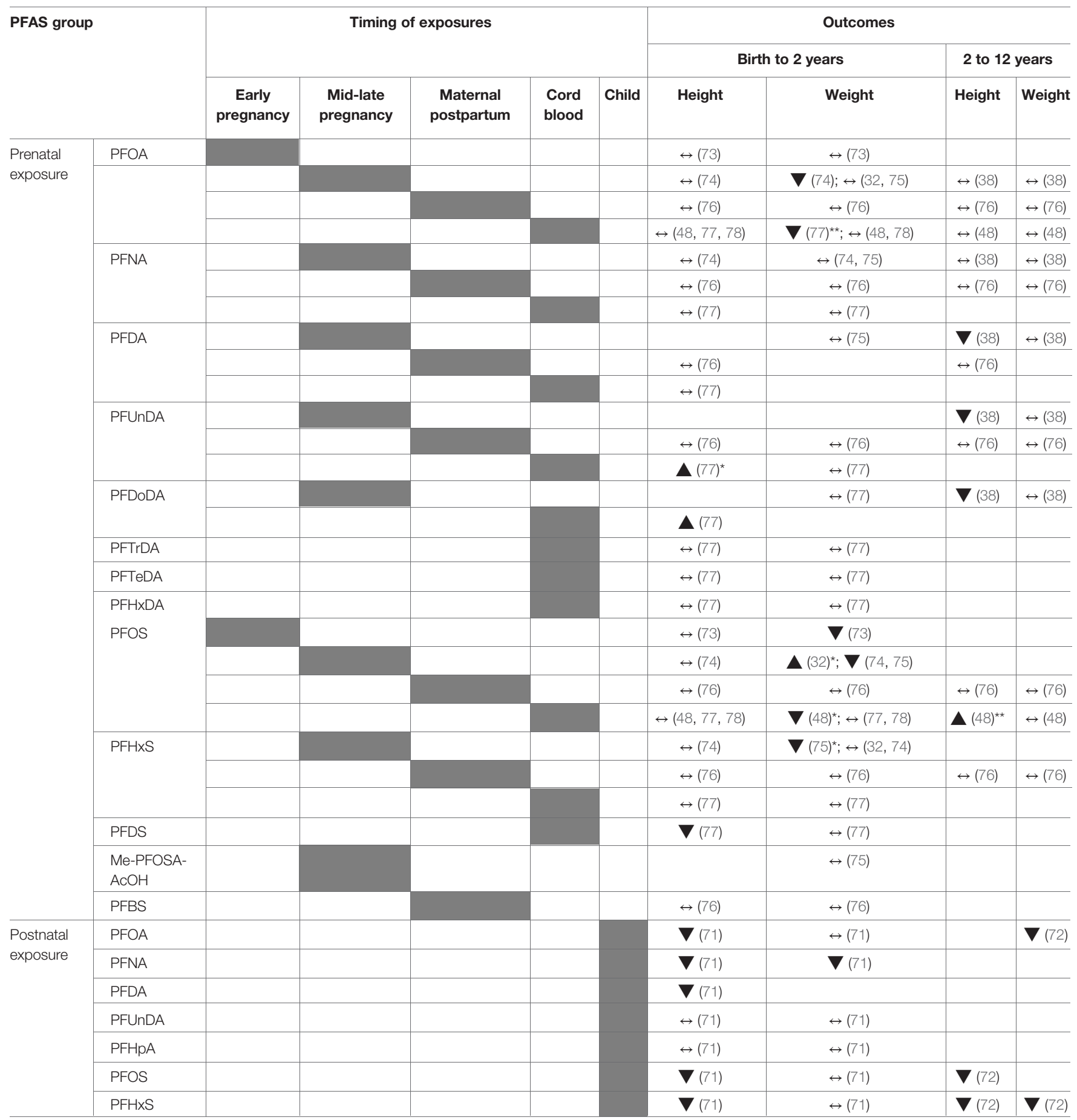

PFOA, perfluorooctanoic acid; PFNA, perfluorononanoic acid; PFDA, perfluorodecanoic acid; PFUnDA, perfluoroundecanoic acid; PFDoDA, perfluorododecanoic acid; PFTrDA, perfluorotridecanoic acid; PFTeDA, perfluorotetradecanoic acid; PFHXDA, perfluorohexadecanoic acid; PFOS, perfluorooctane sulfonic acid; PFHXS, perfluorohexane sulfonic acid; PFDS, perfluorodecane sulfonic acid; Me-PFOSA-AcOH, N-methyl-perfluorooctane sulfonamidoacetic acid; PFBS, perfluorobutane sulfonic acid; PFHpA, perfluoroheptanoic acid

$\boldsymbol{\Lambda}$, Positive associations; $\mathbf{\nabla}$, Inverse association; $\leftrightarrow$, Null association; * significant only in girls; ** significant only in boys.

Early and mild-late pregnancy refer to $1^{\text {st }}$ trimester, and $2^{\text {nd }}$ or $3^{\text {rd }}$ trimester, respectively.

Long-chain PFAS include PFOA, PFNA, PFDA, PFUnDA, PFDODA, PFTrDA, PFTeDA and PFHXDA in PFCA group, and PFOS, PFHXS, PFDS, and Me-PFOSA-AcOH in PFSA group. Short-chain PFAS include PFHDA in PFCA group and PFBS in PFSA group.

Shading boxes indicate exposure timing. 
maternal serum PFOS and weight in girls at 20 months of age (32), however, the positive association was only significant in those born in the lower and upper birth weight stratums and not in those of the middle birthweight stratum. For all other prenatal PFAS including PFDA, PFUnDA, and PFHxS, there were no significant associations in terms of changes in weight in children before 2 years of age. However, for postnatal PFAS exposures, a cross sectional analysis of 2-year-old children found that PFOA and PFNA levels were inversely associated with weight in both sexes, although only PFNA was significant after adjustment (71). For postnatal weight in childhood past 2 years, three studies $(38,48,76)$ demonstrated no associations between all prenatal PFAS exposures and childhood weight, while one cross-sectional studies showed inverse associations between PFOA and PFHxS with weight $\mathrm{z}$-scores between 3-11 years of age (72).

Weight measures demonstrated more significant associations with prenatal and/or postnatal PFAS exposures, particularly in the first 2 years of life. When the results of studies that assessed for PFOA and PFOS exposures are taken together, about half of the studies show associations to weight parameters that are statistically significant. In the majority of these studies, the direction of the associations was largely negative, especially for PFOS. The evidence suggests that PFAS may have either a null effect or a negative on weight within the first two years of life, without definite associations with weight parameters after 2 years of age.

\section{Postnatal Adiposity}

We reviewed 23 studies that examined the relationship of PFAS with measures of adiposity in infancy, childhood, adolescence and early adulthood (Table 3 and Supplementary Table 4). Outcome measures varied according to age and included the ponderal index (SDS), weight gain (change in weight $\mathrm{z}$-scores), body mass index (BMI, z-scores), waist circumference (z-scores, waist-to-height ratio, and/or waist-hip ratio), skinfold thickness, and fat mass as a percentage of total body mass (by air displacement plethys mography, dual-energy X-ray absorptiometry or bioelectric impedance). Prenatal exposures to PFAS were measured in maternal samples taken during gestation or shortly afterwards and in cord blood at birth. Five studies were cross-sectional, and assessed exposure and outcomes in children and adolescents between the ages of 7 and 21 years. PFOA and PFOS were the most frequently analyzed and included in all studies reviewed. More than half of the reviewed studies also analyzed for the effects of other PFAS including PFNA, PFDA, and PFHxS. PFAS that were included in some of the studies were PFDA, PFDoDA, PFHpS, PFOSA, Me-PFOSA-AcOH, PFHpA, and PFBS.

For infant adiposity before 2 years of age, six studies assessed various adiposity measures in infants ranging from 3 to 18 months, including the BMI $(48,74,79,91)$, ponderal index (79), trajectory of BMI changes (83), waist circumference (79), and body fat mass (75, $79,91)$. For studies reporting BMI outcomes, the association to prenatal PFOA was mostly inverse $(74,83)$ or null $(48,91)$, except for one study (79). Repeated anthropometric measures between 4 weeks and 2 years showed monotonic decreases in BMI z-scores of 0.14 and 0.36 in the second and third tertiles of maternal PFOA (74) and analysis of the BMI trajectory showed that the infancy BMI zenith was lower in magnitude in the highest tertile of PFOA (83). Similarly, inverse associations between BMI and PFOS were reported in the same studies $(74,83)$ as well as by Chen et al., who reported decreases in BMI between 6 and 24 months in girls with increasing PFOS (48). These findings, taken together with the generally inverse associations to weight at birth and in early life, may support evidence of continued negative effects on weight by prenatal PFAS exposures. However, inconsistencies remain, as PFOS was positively associated with BMI and the risk of being overweight at 18 months of age (91), and PFOA was also positively associated with other measures of adiposity $(75,79)$. PFOA, along with PFNA and PFDA showed a positive association with the ponderal index in infant girls (79), and increased PFOA and PFNA were related to gains in fat mass percentage in infant boys at 5 months (75).

For BMI measures in early and mid-childhood, more than half of the studies showed positive associations with prenatal PFOA exposures. Maternal and cord blood PFOA was positively associated with higher risk of being overweight between 5 and 9 years of age $(84,86,91)$, greater waist-to-height ratio $(85,86,88)$, and/or BMI zscores $(76,84)$ in childhood. Most of the studies that showed positive associations were those that had assessed PFOA in mid to late pregnancy, while those studies that had assessed early pregnancy PFOA showed null associations with adiposity in childhood. Only one study from the ALSPAC cohort showed inverse associations between prenatal PFOA exposures and both BMI and waist circumference in girls (87). Maternal PFOS was also modestly associated with increased BMI z-scores $(76,80,84)$, waistto-height ratio (86), skinfold thickness (84) and increased risk of being overweight/obese in childhood (84). However, other studies reported null associations with maternal PFOS $(80-82,85,86,88$, $89,91,92)$, and one study showed inverse associations with BMI and waist circumferences in girls (87). For other prenatal PFAS exposures, $\mathrm{PFHxS}$ was associated with increases in the waist-to-hip ratio and the risk of overweight/obesity (88). One study from China demonstrated a positive association of PFBS, but inverse association of PFDoDA with waist circumference, waist-to-height ratio, body fat mass and body fat percentage at 5 years of age (92). For childhood exposures of PFAS and adiposity measures, one study reported consistently decreased BMI and waist-to-height ratios in 7year old children with increased PFOA, PFDA, PFOS, and MePFOSA-AcOH concentrations (94), however, other studies showed null associations $(88,95)$.

While many studies reported positive associations of prenatal exposures PFOA and PFOS exposures with adiposity measures, nonmonotonic dose-responses were noted in two studies of the HOME prospective cohort between maternal PFOA levels and adiposity measures $(83,85)$. Measures of BMI, waist circumference and body fat between 2 and 8 years increase up to the second tertile, followed by declines in the third tertile of PFOA (85). BMI trajectories from 4 weeks to 12 years of age showed monotonic inverse associations with PFOS and PFHxS, null associations with other prenatal PFAS, but non-monotonic dose-responses to PFOA: the timing and magnitude of the infancy BMI zenith was similar in the first and second tertiles of PFOA, followed by more rapid increases to a higher BMI between 8 and 12 years in the second tertile. The third tertile, in comparison, had an earlier and lower magnitude of the infancy BMI peak, followed by 
TABLE 3 | Summary of studies assessing associations between PFAS exposure and adiposity outcomes.

\begin{tabular}{|c|c|c|c|c|c|c|c|c|c|c|c|c|c|c|c|c|}
\hline \multicolumn{2}{|c|}{ PFAS group } & \multicolumn{5}{|c|}{ Timing of exposures } & \multicolumn{10}{|c|}{ Outcomes } \\
\hline & & \multicolumn{2}{|c|}{ Pregnancy } & \multirow{2}{*}{$\begin{array}{c}\text { Maternal } \\
\text { postpartum }\end{array}$} & \multirow{2}{*}{$\begin{array}{l}\text { Cord } \\
\text { blood }\end{array}$} & \multirow[t]{2}{*}{ Child } & \multicolumn{3}{|c|}{ Birth to 2 years } & \multicolumn{4}{|c|}{2 to 12 years } & \multicolumn{3}{|c|}{$13-20$ years } \\
\hline & & Early & Mid-late & & & & BMI/PI & wc & $\mathrm{BF} \%$ & BMI & wc & SFT & $\mathrm{BF} \%$ & BMI & wc & SFT \\
\hline \multirow[t]{23}{*}{$\begin{array}{l}\text { Prenatal } \\
\text { exposure }\end{array}$} & \multirow[t]{4}{*}{ PFOA } & & & & & & $\boldsymbol{\Delta}(79)^{*}$ & $\stackrel{\leftrightarrow}{(79)}$ & $\leftrightarrow(79)$ & $\leftrightarrow(80-82)$ & $\leftrightarrow(80-82)$ & $\leftrightarrow(80)$ & $\leftrightarrow(80)$ & & & \\
\hline & & & & & & & $\boldsymbol{\nabla}(74,83)$ & & $\boldsymbol{\Delta}(75)^{\star \star}$ & 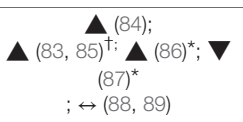 & $\underset{\underset{*}{\boldsymbol{\nabla}}(87)}{(85,88)^{\dagger} ; \boldsymbol{\Delta}}(86)^{\star}$ & $\underset{(84)}{\Delta}$ & $\begin{array}{c}\boldsymbol{\Delta}(85)^{\dagger} ; \leftrightarrow(88) \\
\quad ; \leftrightarrow(87)^{\star}\end{array}$ & $\boldsymbol{\Delta}(90)^{*}$ & $\boldsymbol{\Delta}(90)^{*}$ & \\
\hline & & & & & & & $\leftrightarrow(91)$ & & & $\mathbf{\Delta}(76,91)$ & & & & & & \\
\hline & & & & & & & $\leftrightarrow(48)$ & & & $\leftrightarrow(48,88,92)$ & $\boldsymbol{\Delta}(88) ; \leftrightarrow(92)$ & & $\leftrightarrow(88,92)$ & & & \\
\hline & \multirow[t]{4}{*}{ PFNA } & & & & & & $\boldsymbol{\Delta}(79)^{*}$ & $\stackrel{\leftrightarrow}{(79)}$ & $\boldsymbol{\Delta}(79)^{*}$ & $\leftrightarrow(80,82)$ & $\leftrightarrow(80,82)$ & $\underset{(80)^{\star}}{\boldsymbol{\Delta}}$ & $\leftrightarrow(80)$ & & & \\
\hline & & & & & & & $\leftrightarrow(74,83)$ & & $\boldsymbol{\Delta}(75)^{\star \star}$ & $\stackrel{(83,85,88,89) ; \leftrightarrow}{(87)^{*}}$ & $\leftrightarrow(85,88) ; \leftrightarrow(87)^{\star}$ & & $\begin{array}{c}\leftrightarrow(85,88) ; \leftrightarrow \\
\quad(87)^{\star}\end{array}$ & $\leftrightarrow(90)$ & $\leftrightarrow(90)$ & \\
\hline & & & & & & & $\leftrightarrow(91)$ & & & $\boldsymbol{\Delta}(76) ; \leftrightarrow(91)$ & & & & & & \\
\hline & & & & & & & & & & $\leftrightarrow(88,92)$ & $\leftrightarrow(88,92)$ & & $\leftrightarrow(88,92)$ & & & \\
\hline & \multirow[t]{4}{*}{ PFDA } & & & & & & $\boldsymbol{\Delta}(79)^{*}$ & $\stackrel{\leftrightarrow}{(79)}$ & $\boldsymbol{\Delta}(79)^{*}$ & & & & & & & \\
\hline & & & & & & & & & $\leftrightarrow(75)$ & & & & & & & \\
\hline & & & & & & & $\leftrightarrow(91)$ & & & $\leftrightarrow(76,91)$ & & & & & & \\
\hline & & & & & & & & & & $\leftrightarrow(92)$ & $\leftrightarrow(92)$ & & $\leftrightarrow(92)$ & & & \\
\hline & PFUnDA & & & & & & & & & $\leftrightarrow(76)$ & & & & & & \\
\hline & PFDoDA & & & & & & & & & $\begin{array}{l}\leftrightarrow(92) \\
\leftrightarrow(92)\end{array}$ & $\leftrightarrow_{\boldsymbol{\nabla}}^{(92)}$ & & $\stackrel{\leftrightarrow}{\nabla}_{(92)}^{(92)}$ & & & \\
\hline & \multirow[t]{4}{*}{ PFOS } & & & & & & $\leftrightarrow(79)$ & $\stackrel{\leftrightarrow}{(79)}$ & $\leftrightarrow(79)$ & $\leftrightarrow(80-82,86)$ & $\leftrightarrow(80-82)$ & $\leftrightarrow(80)$ & $\leftrightarrow(80)$ & & & \\
\hline & & & & & & & $\boldsymbol{\nabla}(74,83)$ & & $\leftrightarrow(75)$ & $\begin{array}{c}\boldsymbol{\Delta}(84) ; \boldsymbol{\nabla}(83) ; \boldsymbol{\nabla}(87)^{\star} \\
; \leftrightarrow(85,86,88,89)\end{array}$ & $\begin{aligned} & \boldsymbol{\Delta}(86)^{\star} ; \boldsymbol{\nabla}(87)^{\star} \\
& ; \leftrightarrow(85,88)\end{aligned}$ & $\underset{(84)}{\boldsymbol{\Lambda}}$ & $\begin{array}{c}(85,88) ; \leftrightarrow \\
(87)^{\star}\end{array}$ & $\leftrightarrow(90)$ & $\leftrightarrow(90)$ & \\
\hline & & & & & & & $\boldsymbol{\Delta}(91)$ & & $\leftrightarrow(91)$ & $\mathbf{\Delta}(76)$ & & & & & & \\
\hline & & & & & & & $\boldsymbol{\nabla}(48)^{*}$ & & & $\underset{; \leftrightarrow(88,92)}{\boldsymbol{\Delta}(48)^{*}}$ & $\leftrightarrow(88,92)$ & & $\leftrightarrow(88,92)$ & & & \\
\hline & \multirow[t]{3}{*}{ PFHXS } & & & & & & $\leftrightarrow(79)$ & $\stackrel{\leftrightarrow}{(79)}$ & $\leftrightarrow(79)$ & $\leftrightarrow(80,82)$ & $\leftrightarrow(80,82)$ & $\underset{(80)^{\star}}{\boldsymbol{\Delta}}$ & $\leftrightarrow(80)$ & & & \\
\hline & & & & & & & $\begin{array}{l}\boldsymbol{\nabla}(83) \\
\leftrightarrow(74)\end{array}$ & & $\leftrightarrow(75)$ & $\begin{aligned} \boldsymbol{\nabla}(83) ; & \leftrightarrow(85,88,89) ; \\
& \leftrightarrow(87)^{\star}\end{aligned}$ & $\leftrightarrow(85,88) ; \leftrightarrow(87)^{\star}$ & & $\underset{(87)^{\star}}{\boldsymbol{\Delta}(88) ; \leftrightarrow(85) ; \leftrightarrow}$ & & & \\
\hline & & & & & & & $\leftrightarrow(91)$ & & & $\boldsymbol{\Delta}(76) ; \leftrightarrow(91)$ & & & & & & \\
\hline & PFBS & & & & & & & & & $\begin{aligned} \leftrightarrow & (88,92) \\
& \leftrightarrow(92)\end{aligned}$ & $\begin{array}{c}\boldsymbol{\Delta}(88) ; \leftrightarrow(92) \\
\quad \mathbf{\Delta}(92)\end{array}$ & & $\begin{array}{c}\leftrightarrow(88,92) \\
\quad \mathbf{\Delta}(92)\end{array}$ & & & \\
\hline & $\begin{array}{l}\text { PFOSA } \\
\text { Me-PFOSA- } \\
\text { ACOH }\end{array}$ & & & & & & & & $\leftrightarrow(75)$ & & & & & $\leftrightarrow(90)$ & $\leftrightarrow(90)$ & \\
\hline
\end{tabular}




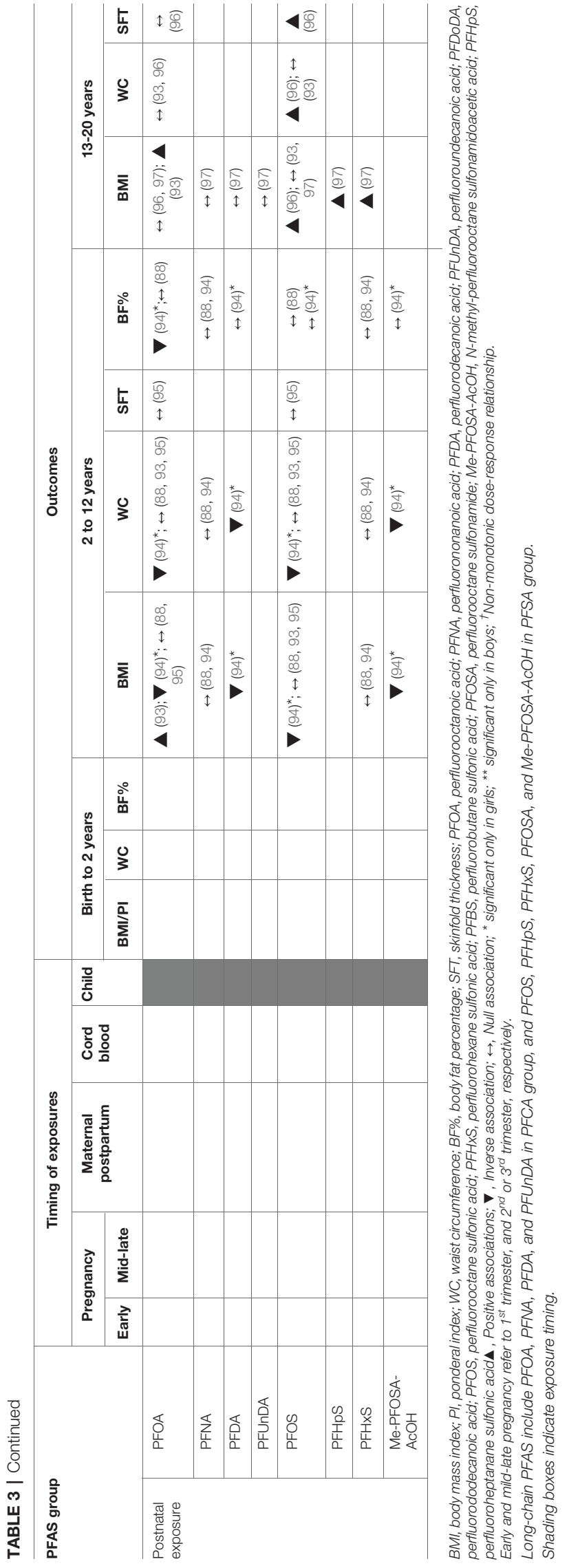

increases in absolute BMI from 8 to 12 years that were similar to the first tertile of PFOA (83).

For adiposity measures in adolescence (past 12 years of age) and young adulthood, only four studies were present including two longitudinal cohort studies that assessed exposures in maternal blood (90) or during childhood (96) and two crosssectional study during adolescence $(93,97)$. Both longitudinal cohort studies showed positive associations, with Halldorsson et al., demonstrating a positive association between maternal PFOA and offspring BMI, waist circumference, and the risk of being overweight at 20 years of age (in females) (90) and Domazet et al., demonstrating that PFOS exposure at 9 years of age was positively associated with adiposity measures at 15 years (BMI, skinfold thickness and waist circumference) and at 21 years (skinfold thickness) of age (96). For cross-sectional studies, one study from NHANES showed an increased risk of overweight and/or obesity with higher PFOA exposures but not with PFOS exposures in adolescents aged 12 to 18 years (93). One other study demonstrated no definite associations between PFOA and PFOS and adolescent BMI, but significant positive relationships of PFHxS and PFHpS with the presence of overweight and obesity in adolescents aged 15 to 19 years (97).

In summary, studies of adiposity in infancy mostly support evidence for continued inverse effects of PFAS on BMI in the first 2 years of life. However, there are inconsistencies in the results, reflecting the complexities of analyzing adiposity during infancy, a time characterized by dynamic physiologic changes in weight and height (98). Studies of childhood and adolescent adiposity were split between either null or positive associations with different PFAS. For those studies that did report significant associations, the direction of the association were mostly positive for PFOA, with similar but more modest findings in regards to PFOS.

\section{Puberty, Menarche, and Sex Hormone Levels}

Puberty is a time of transition, characterized by physiological and psychological changes, to achieve sexual maturation and fertility. The intrauterine milieu, birth size, nutrition, and endocrine disrupting chemicals (EDCs) may affect adiposity trajectory, pubertal timing and progression, with adiposity itself also contributing to changes in puberty and sex hormone levels (99-101). The relationships of PFAS exposures with pubertal onset, menarche, and sex hormone levels during childhood and adolescence have been evaluated in eleven studies (Table $\mathbf{4}$ and Supplementary Table 5). The outcome measures included selfassessed pubertal stage, age at menarche, and levels of luteinizing hormone ( $\mathrm{LH})$, follicle stimulating hormone (FSH), testosterone, estradiol, and sex hormone-binding globulin (SHBG).

With regards to girls' pubertal development and menarche, five studies showed inconsistent results (Table 4) $(102-105,108)$. For pubertal development, the Danish National Birth Cohort (DNBC) study recently reported substance-specific and non-monotonic associations between prenatal PFAS exposure and onset of pubertal indicators in girls (102). In detail, prenatal exposures to PFNA, PFDA, PFOS, PFHpS, and PFHxS were associated with earlier age at onset for pubertal milestones, with non-monotonic 
TABLE 4 | Summary of studies assessing associations between PFAS exposure and puberty, menarche, and sex hormone levels.

PFAS group Timing of exposures

Outcomes

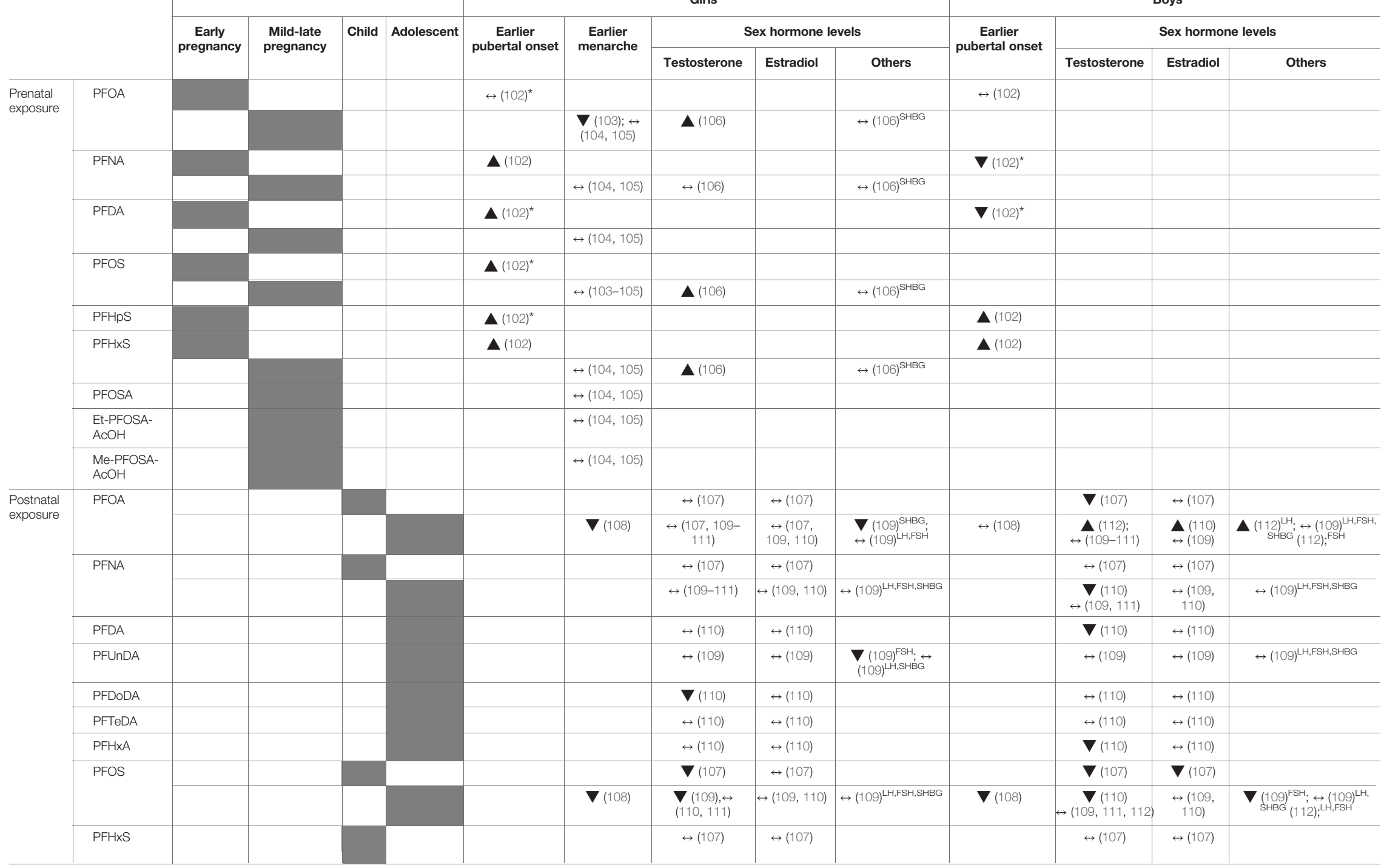




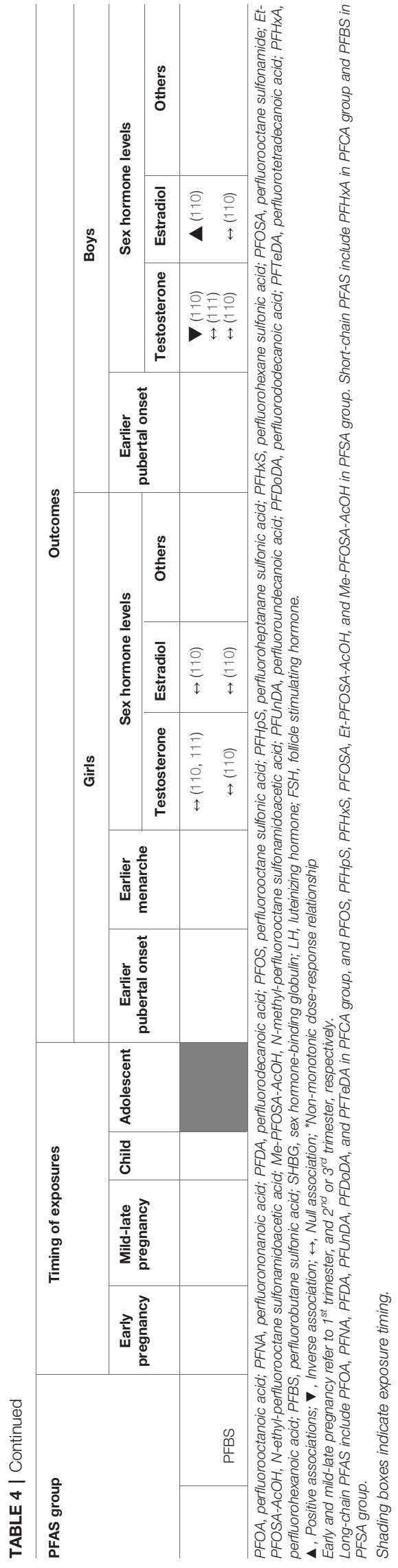

dose- responses for PFDA, PFOS, and PFHpS showing earlier pubertal indicators in the middle rather than in the highest exposure tertiles, with the lowest as a reference. For age at menarche, three prospective studies evaluated maternal exposure during pregnancy (103-105) and one cross-sectional study investigated postnatal exposure during adolescence (108). The DNBC study (103) reported later age of menarche with higher levels of prenatal PFOA exposure, while data from the Avon Longitudinal Study of Parents and Children (ALSPAC) cohort showed no associations between prenatal PFAS exposures and age at menarche $(104,105)$. On the contrary, the C8 Health project from United States reported a cross-sectional relationship in which higher concentrations of PFOA and PFOS were related to decreased odds of postmenarche, suggesting a later age of puberty in adolescent girls (108).

For girls' hormone levels, five studies evaluated the relationship between PFAS exposures and testosterone, estradiol, and gonadotropin levels in girls, and the results were inconsistent (106, 107, 109-111). The ALSPAC cohort study showed positive associations between prenatal exposures to PFOA, PFOS, and PFHxS and serum testosterone levels in 15-year-old girls (106). On the contrary, three cross-sectional studies found inverse associations between postnatal PFOS $(107,109)$ or PFDoDA exposures (110) and serum testosterone levels in 6 to 9-year-old girls from the C8 Health project (107) or adolescent girls from Taiwan $(109,110)$. A cross-sectional study using data from NHANES (2011-2012) from the United States reported no relationships between PFAS exposures and testosterone levels in girls (111). Three studies showed no relationships between PFAS exposures and estradiol levels in girls during childhood and adolescence $(107,109,110)$. One study reported inverse associations between PFAS exposures with FSH or SHBG levels in adolescent girls (109).

For boys' pubertal indicators, two studies showed inconsistent results according to the timing of exposure (prenatal or postnatal) $(102,108)$. The aforementioned DNBC study (102) also reported substance-specific and non-monotonic associations between prenatal PFAS exposures and pubertal development in boys. In detail, prenatal PFHpS, and PFHxS exposures were associated with younger age at onset for pubertal milestones, whereas prenatal PFNA and PFDA exposures were related to later age of pubertal indicators in boys, with non-monotonous associations for PFNA and PFDA. On the contrary, the C8 Health project reported a cross-sectional relationship of reduced odds of reached puberty assessed by testosterone levels (>50 ng/dL) with increasing PFOS levels in boys (108).

For boys' hormone levels, five cross-sectional studies have been reported (107, 109-112). Postnatal exposure to PFOA, PFNA, PFDA, PFOS, or PFHXA showed inverse associations with serum testosterone levels $(107,110)$, positive associations (112), or null associations $(109,111)$. The cross-sectional relationships between PFAS exposures and estradiol levels were also inconsistent; positive (110), inverse (107), or null (109) according to the subjects' age or type of PFAS. One study reported inverse associations between PFAS exposures and FSH levels in adolescent boys (109).

Overall, pediatric studies that evaluated the associations between early-life PFAS exposures and pubertal development 
or sex hormone levels were limited with inconsistent results according to timing, level of exposure, and type of PFAS.

\section{DISCUSSIONS}

In this review, we focused on the effects of prenatal to postnatal PFAS exposures on growth, adiposity, and puberty in children and adolescents. Contrary to relatively sufficient evidence on fetal growth and childhood adiposity, few studies have been conducted on childhood growth and puberty. Furthermore, the results were inconsistent according to sex, timing of exposure, type of PFAS, and levels of exposure. For birth size, prenatal PFAS exposure may mostly impair fetal growth. For growth within the first 2 years, prenatal PFAS exposure exhibited no association with height, but null or negative relationships with weight. However, postnatal PFAS exposure showed inverse relationship with height and weight at 2 years in a cross-sectional study. For postnatal adiposity, prenatal PFAS may mostly have negative impact on BMI in the first 2 years of life, but a positive effect on childhood and adolescent adiposity, although some studies showed no associations. For pubertal development or sex hormone levels, the evidence was limited and inconclusive.

Although mechanisms through which PFAS affect early-life growth, adiposity, and pubertal development in humans remain unclear, experimental studies have proposed potential modes of action. For prenatal and postnatal growth, one possible mechanism of PFAS toxicity is PFAS-mediated activation of the peroxisome proliferator-activated receptor $\alpha(\operatorname{PPAR} \alpha)$ or PPAR $\gamma$ (113). Prenatal PFAS-activated PPAR signaling may alter placental and fetal metabolic tissue development, leading to impairments in fetal growth (114). In mouse models, PFOA-induced developmental toxicity and growth impairment in utero occurred in the presence of PPAR $\alpha$ activity (115). Although most studies have focused on the effects of PFOA or PFOS, a recent in-vitro study demonstrated the human PPAR activating potential of various PFAS substances including short-chain PFAS (116). PPARs were also shown to be involved in modulating glucose and lipid metabolism or adipocyte differentiation (117). Recent epidemiologic studies showing inverse associations between maternal PFAS exposures and fatty acids during pregnancy (36) suggest a negative impact of prenatal PFAS exposures on birth weight, through reduced availability of maternal fatty acids in utero. Meanwhile, PFAS-induced activation of PPAR $\gamma$ can lead to adipogenesis and inflammation (118), contributing to increased adiposity and risk of obesity in children and adolescents.

PFAS have been identified as thyroid disrupting chemicals, potentially affecting hypothalamic-pituitary-thyroid axis, thyroid hormone synthesis and metabolism $(119,120)$. Since thyroid hormones play a crucial role in normal growth and development, altered thyroid function can affect early-life growth and adiposity during critical periods of development. There is a possibility that the effect of PFAS on early-life growth can be mediated by thyroid hormone disruption, although a recent study did not provide evidence of the mediating role of thyroid hormones (63). In addition, some PFAS can interfere with steroid hormone synthesis and metabolism. PFAS can inhibit 11- $\beta$ hydroxysteroid dehydrogenase 2 with subsequent increases in glucocorticoid concentrations (121), leading to alterations in placental development and function, and impairment of fetal growth (122). PFOS suppresses estradiol, progesterone, and human chorionic gonadotropin secretion by placental syncytiotrophoblasts (123), which may also disrupt normal placental development and function.

For pubertal development, PFAS can affect the hypothalamicpituitary-gonadal axis (124). PFAS can also directly affect gonads through their weak estrogenic or anti-androgenic actions (125) and perturb pubertal development. Neonatal and juvenile exposure to PFOA and PFOS in female rats altered gene expression in the kisspeptin system in the hypothalamus, leading to advanced pubertal onset (126). The effect of PFAS on childhood adiposity (127) can trigger the metabolic and peripheral signals linked to pubertal development (128). Also, PFAS can affect adrenal or gonadal steroidogenesis through altering enzyme activity $(121,129,130)$.

Although experimental animal studies have suggested various plausible mechanisms, evidence in humans is still lacking. Current human evidence on the impact of PFAS exposures on childhood growth, adiposity, and puberty are limited with inconsistent data. The reasons for the discrepancies in human studies are complex, probably due to various factors, including the variety of PFAS in the environment, the developmental timewindow of exposure, concomitant exposure to chemical mixtures, and the possibility of non-monotonic dose-response relationships. This complexity renders it difficult to build a reliable epidemiological model to fully reveal the mechanisms of EDC actions and to determine the real clinical impact of EDC on human health outcomes (131).

PFAS are comprised of more than 4000 individual compounds, however, previous studies mainly focused on long-chain PFAS, especially PFOA or PFOS. With the restrictions on the use of long-chain PFAS due to their persistence, bioaccumulation potential, and toxicity, they have been mainly replaced by structurally similar short-chain or other novel PFAS (132). New generation PFAS are expected to have lower toxicities due to shorter half-lives. However, the increased use of short-chain PFAS has also generated concerns regarding childhood health effects, since they have high water solubility, resistance to degradation from the environment, and modes of action similar to long-chain PFAS (7, 133). Additionally, cumulative or interactive toxicities of PFAS as mixtures remain unknown, since most studies have examined PFAS as individual compounds. A few in vitro studies revealed that the interactions between PFAS compounds can be complicated, as they can act additively or interact synergistically or antagonistically, depending on the species, dose ranges, duration of exposure, and mixture components (134). As humans are exposed to complex mixtures of PFAS in daily life, the combined effects or potential interactions of multiple PFAS need to be further clarified (135). Moreover, non-monotonic dose-responses, defined as a nonlinear relationship between dose and effect, are common in studies of hormones and EDCs (136). The more 
disruptive effect of PFAS at medium dose levels than high dose levels has been identified for childhood adiposity $(83,85)$ and puberty (102). Future investigation on the relationship between PFAS and health effects should include assessment of nonmonotonic dose-response relationships with appropriate statistical modelling. In terms of outcomes, dynamic changes and trajectories of childhood growth, adiposity, and puberty need to be further studied with studies of prospective and longitudinal design.

\section{CONCLUSIONS}

Early-life exposure to PFAS during critical periods of development may affect fetal and postnatal growth, adiposity, and pubertal development, potentially leading to latent health effects in adulthood. The current epidemiologic evidence has mostly suggested that prenatal PFAS exposures may impair fetal growth and increase childhood adiposity, although data on the effects of early-life PFAS exposures on childhood growth or pubertal development are still limited and inconsistent. The mechanisms through which PFAS affect early-life physical development in humans remain unclear, although experimental animal studies have suggested potential modes of action including PFAS-induced PPAR activation, altered thyroid and steroid hormone synthesis and metabolism, and weak estrogenic or anti-androgenic activity. Further research, designed to evaluate the various types of PFAS

\section{REFERENCES}

1. Lindstrom AB, Strynar MJ, Libelo EL. Polyfluorinated Compounds: Past, Present, and Future. Environ Sci Technol (2011) 45(19):7954-61. doi: 10.1021/es2011622

2. Giesy JP, Kannan K. Global Distribution of Perfluorooctane Sulfonate in Wildlife. Environ Sci Technol (2001) 35(7):1339-42. doi: 10.1021/es001834k

3. Kannan K, Corsolini S, Falandysz J, Fillmann G, Kumar KS, Loganathan BG, et al. Perfluorooctanesulfonate and Related Fluorochemicals in Human Blood From Several Countries. Environ Sci Technol (2004) 38(17):448995. doi: 10.1021/es0493446

4. Buck RC, Franklin J, Berger U, Conder JM, Cousins IT, de Voogt P, et al. Perfluoroalkyl and Polyfluoroalkyl Substances in the Environment: Terminology, Classification, and Origins. Integr Environ Assess Manag (2011) 7(4):513-41. doi: 10.1002/ieam.258

5. Kato K, Wong LY, Jia LT, Kuklenyik Z, Calafat AM. Trends in Exposure to Polyfluoroalkyl Chemicals in the U.S. Population: 1999-2008. Environ Sci Technol (2011) 45(19):8037-45. doi: 10.1021/es1043613

6. Butenhoff JL, Olsen GW, Pfahles-Hutchens A. The Applicability of Biomonitoring Data for Perfluorooctanesulfonate to the Environmental Public Health Continuum. Environ Health Perspect (2006) 114(11):177682. doi: $10.1289 /$ ehp. 9060

7. Ateia M, Maroli A, Tharayil N, Karanfil T. The Overlooked Short- and Ultrashort-Chain Poly- and Perfluorinated Substances: A Review. Chemosphere (2019) 220:866-82. doi: 10.1016/j.chemosphere.2018.12.186

8. Olsen GW, Burris JM, Ehresman DJ, Froehlich JW, Seacat AM, Butenhoff JL, et al. Half-Life of Serum Elimination of Perfluorooctanesulfonate, Perfluorohexanesulfonate, and Perfluorooctanoate in Retired Fluorochemical Production Workers. Environ Health Perspect (2007) 115 (9):1298-305. doi: 10.1289/ehp.10009

9. Sunderland EM, Hu XC, Dassuncao C, Tokranov AK, Wagner CC, Allen JG. A Review of the Pathways of Human Exposure to Poly- and Perfluoroalkyl as mixtures, and in consideration of dynamic growth and adiposity trajectories, the critical time-window of exposure, and possible nonmonotonic dose-response relationships, is warranted.

\section{AUTHOR CONTRIBUTIONS}

YL, HK, HJ, and YC performed the literature search, and wrote and revised the manuscript. YL conceptualized and coordinated the study, and critically revised the manuscript. All authors contributed to the article and approved the submitted version.

\section{FUNDING}

This work was supported by the National Research Foundation of Korea (NRF) grant funded by the Korea government (MSIT) (No. 2018R1D1A1B07049806). This study was also supported by the Seoul National University Hospital Research Fund (No. 042018-3030).

\section{SUPPLEMENTARY MATERIAL}

The Supplementary Material for this article can be found online at: https://www.frontiersin.org/articles/10.3389/fendo.2021. 683297/full\#supplementary-material

Substances (PFASs) and Present Understanding of Health Effects. J Expo Sci Environ Epidemiol (2019) 29(2):131-47. doi: 10.1038/s41370-018-0094-1

10. Gutzkow KB, Haug LS, Thomsen C, Sabaredzovic A, Becher G, Brunborg G. Placental Transfer of Perfluorinated Compounds is Selective-a Norwegian Mother and Child Sub-Cohort Study. Int J Hyg Environ Health (2012) 215 (2):216-9. doi: 10.1016/j.ijheh.2011.08.011

11. Mondal D, Lopez-Espinosa MJ, Armstrong B, Stein CR, Fletcher T. Relationships of Perfluorooctanoate and Perfluorooctane Sulfonate Serum Concentrations Between Mother-Child Pairs in a Population With Perfluorooctanoate Exposure From Drinking Water. Environ Health Perspect (2012) 120(5):752-7. doi: 10.1289/ehp.1104538

12. Rappazzo KM, Coffman E, Hines EP. Exposure to Perfluorinated Alkyl Substances and Health Outcomes in Children: A Systematic Review of the Epidemiologic Literature. Int J Environ Res Public Health (2017) 14(7):691. doi: 10.3390/ijerph14070691

13. Barker DJ, Osmond C. Infant Mortality, Childhood Nutrition, and Ischaemic Heart Disease in England and Wales. Lancet (1986) 1 (8489):1077-81. doi: 10.1016/s0140-6736(86)91340-1

14. Moher D, Liberati A, Tetzlaff J, Altman DG, Group P. Preferred Reporting Items for Systematic Reviews and Meta-Analyses: The PRISMA Statement. PloS Med (2009) 6(7):e1000097. doi: 10.1371/journal.pmed.1000097

15. Bero L, Chartres N, Diong J, Fabbri A, Ghersi D, Lam J, et al. The Risk of Bias in Observational Studies of Exposures (ROBINS-E) Tool: Concerns Arising From Application to Observational Studies of Exposures. Syst Rev (2018) 7 (1):242. doi: 10.1186/s13643-018-0915-2

16. Univeristy of Bristol. The ROBINS-E Tool (Risk Of Bias In Non-Randomized Studies - of Exposures). (2021). Available at: http://www.bristol.ac.uk/ population-health-sciences/centres/cresyda/barr/riskofbias/robins-e/ (Accessed May 22, 2021).

17. Fei C, McLaughlin JK, Tarone RE, Olsen J. Perfluorinated Chemicals and Fetal Growth: A Study Within the Danish National Birth Cohort. Environ Health Perspect (2007) 115(11):1677-82. doi: 10.1289/ehp.10506 
18. Meng Q, Inoue K, Ritz B, Olsen J, Liew Z. Prenatal Exposure to Perfluoroalkyl Substances and Birth Outcomes; An Updated Analysis From the Danish National Birth Cohort. Int J Environ Res Public Health (2018) 15(9):1832. doi: 10.3390/ijerph15091832

19. Hjermitslev MH, Long M, Wielsøe M, Bonefeld-Jørgensen EC. Persistent Organic Pollutants in Greenlandic Pregnant Women and Indices of Foetal Growth: The ACCEPT Study. Sci Total Environ (2020) 698:134118. doi: 10.1016/j.scitotenv.2019.134118

20. Wikstrom S, Lin PI, Lindh CH, Shu H, Bornehag CG. Maternal Serum Levels of Perfluoroalkyl Substances in Early Pregnancy and Offspring Birth Weight. Pediatr Res (2020) 87(6):1093-9. doi: 10.1038/s41390-019-0720-1

21. Monroy R, Morrison K, Teo K, Atkinson S, Kubwabo C, Stewart B, et al. Serum Levels of Perfluoroalkyl Compounds in Human Maternal and Umbilical Cord Blood Samples. Environ Res (2008) 108(1):56-62. doi: 10.1016/j.envres.2008.06.001

22. Bach CC, Bech BH, Nohr EA, Olsen J, Matthiesen NB, Bonefeld-Jorgensen EC, et al. Perfluoroalkyl Acids in Maternal Serum and Indices of Fetal Growth: The Aarhus Birth Cohort. Environ Health Perspect (2016) 124 (6):848-54. doi: 10.1289/ehp.1510046

23. Sagiv SK, Rifas-Shiman SL, Fleisch AF, Webster TF, Calafat AM, Ye X, et al. Early-Pregnancy Plasma Concentrations of Perfluoroalkyl Substances and Birth Outcomes in Project Viva: Confounded by Pregnancy Hemodynamics? Am J Epidemiol (2018) 187(4):793-802. doi: 10.1093/aje/ kwx332

24. Manzano-Salgado CB, Casas M, Lopez-Espinosa MJ, Ballester F, Iniguez C, Martinez D, et al. Prenatal Exposure to Perfluoroalkyl Substances and Birth Outcomes in a Spanish Birth Cohort. Environ Int (2017) 108:278-84. doi: 10.1016/j.envint.2017.09.006

25. Ashley-Martin J, Dodds L, Arbuckle TE, Bouchard MF, Fisher M, Morriset AS, et al. Maternal Concentrations of Perfluoroalkyl Substances and Fetal Markers of Metabolic Function and Birth Weight. Am J Epidemiol (2017) 185(3):185-93. doi: 10.1093/aje/kww213

26. Chen L, Tong C, Huo X, Zhang J, Tian Y, Shanghai Birth C. Prenatal Exposure to Perfluoroalkyl and Polyfluoroalkyl Substances and Birth Outcomes: A Longitudinal Cohort With Repeated Measurements. Chemosphere (2021) 267:128899. doi: 10.1016/j.chemosphere.2020.128899

27. Fei C, McLaughlin JK, Tarone RE, Olsen J. Fetal Growth Indicators and Perfluorinated Chemicals: A Study in the Danish National Birth Cohort. Am J Epidemiol (2008) 168(1):66-72. doi: 10.1093/aje/kwn095

28. Wu K, Xu X, Peng L, Liu J, Guo Y, Huo X. Association Between Maternal Exposure to Perfluorooctanoic Acid (PFOA) From Electronic Waste Recycling and Neonatal Health Outcomes. Environ Int (2012) 48:1-8. doi: 10.1016/j.envint.2012.06.018

29. Lenters V, Portengen L, Rignell-Hydbom A, Jonsson BA, Lindh CH, Piersma $\mathrm{AH}$, et al. Prenatal Phthalate, Perfluoroalkyl Acid, and Organochlorine Exposures and Term Birth Weight in Three Birth Cohorts: MultiPollutant Models Based on Elastic Net Regression. Environ Health Perspect (2016) 124(3):365-72. doi: 10.1289/ehp.1408933

30. Minatoya M, Itoh S, Miyashita C, Araki A, Sasaki S, Miura R, et al. Association of Prenatal Exposure to Perfluoroalkyl Substances With Cord Blood Adipokines and Birth Size: The Hokkaido Study on Environment and Children's Health. Environ Res (2017) 156:175-82. doi: 10.1016/ j.envres.2017.03.033

31. Souza MCO, Saraiva MCP, Honda M, Barbieri MA, Bettiol H, Barbosa F, et al. Exposure to Per- and Polyfluorinated Alkyl Substances in Pregnant Brazilian Women and its Association With Fetal Growth. Environ Res (2020) 187:109585. doi: 10.1016/j.envres.2020.109585

32. Maisonet M, Terrell ML, McGeehin MA, Christensen KY, Holmes A, Calafat AM, et al. Maternal Concentrations of Polyfluoroalkyl Compounds During Pregnancy and Fetal and Postnatal Growth in British Girls. Environ Health Perspect (2012) 120(10):1432-7. doi: 10.1289/ehp.1003096

33. Washino N, Saijo Y, Sasaki S, Kato S, Ban S, Konishi K, et al. Correlations Between Prenatal Exposure to Perfluorinated Chemicals and Reduced Fetal Growth. Environ Health Perspect (2009) 117(4):660-7. doi: 10.1289/ ehp. 11681

34. Hamm MP, Cherry NM, Chan E, Martin JW, Burstyn I. Maternal Exposure to Perfluorinated Acids and Fetal Growth. J Expo Sci Environ Epidemiol (2010) 20(7):589-97. doi: 10.1038/jes.2009.57
35. Whitworth KW, Haug LS, Baird DD, Becher G, Hoppin JA, Skjaerven R, et al. Perfluorinated Compounds in Relation to Birth Weight in the Norwegian Mother and Child Cohort Study. Am J Epidemiol (2012) 175 (12):1209-16. doi: 10.1093/aje/kwr459

36. Kishi R, Nakajima T, Goudarzi H, Kobayashi S, Sasaki S, Okada E, et al. The Association of Prenatal Exposure to Perfluorinated Chemicals With Maternal Essential and Long-Chain Polyunsaturated Fatty Acids During Pregnancy and the Birth Weight of Their Offspring: The Hokkaido Study. Environ Health Perspect (2015) 123(10):1038-45. doi: 10.1289/ehp.1408834

37. Alkhalawi E, Kasper-Sonnenberg M, Wilhelm M, Volkel W, Wittsiepe J. Perfluoroalkyl Acids (PFAAs) and Anthropometric Measures in the First Year of Life: Results From the Duisburg Birth Cohort. J Toxicol Environ Health A (2016) 79(22-23):1041-9. doi: 10.1080/15287394.2016.1219552

38. Wang Y, Adgent M, Su PH, Chen HY, Chen PC, Hsiung CA, et al. Prenatal Exposure to Perfluorocarboxylic Acids (PFCAs) and Fetal and Postnatal Growth in the Taiwan Maternal and Infant Cohort Study. Environ Health Perspect (2016) 124(11):1794-800. doi: 10.1289/ehp.1509998

39. Lauritzen HB, Larose TL, Oien T, Sandanger TM, Odland JO, Van De Bor M, et al. Maternal Serum Levels of Perfluoroalkyl Substances and Organochlorines and Indices of Fetal Growth: A Scandinavian CaseCohort Study. Pediatr Res (2017) 81(1):33-42. doi: 10.1038/pr.2016.187

40. Kashino I, Sasaki S, Okada E, Matsuura H, Goudarzi H, Miyashita C, et al. Prenatal Exposure to 11 Perfluoroalkyl Substances and Fetal Growth: A Large-Scale, Prospective Birth Cohort Study. Environ Int (2020) 136:105355. doi: 10.1016/j.envint.2019.105355

41. Eick SM, Hom Thepaksorn EK, Izano MA, Cushing LJ, Wang Y, Smith SC, et al. Associations Between Prenatal Maternal Exposure to Per- And Polyfluoroalkyl Substances (PFAS) and Polybrominated Diphenyl Ethers (PBDEs) and Birth Outcomes Among Pregnant Women in San Francisco. Environ Health (2020) 19(1):100. doi: 10.1186/s12940-020-00654-2

42. Gardener H, Sun Q, Grandjean P. PFAS Concentration During Pregnancy in Relation to Cardiometabolic Health and Birth Outcomes. Environ Res (2021) 192:110287. doi: 10.1016/j.envres.2020.110287

43. Marks KJ, Cutler AJ, Jeddy Z, Northstone K, Kato K, Hartman TJ. Maternal Serum Concentrations of Perfluoroalkyl Substances and Birth Size in British Boys. Int J Hyg Environ Health (2019) 222(5):889-95. doi: 10.1016/ j.ijheh.2019.03.008

44. Li M, Zeng XW, Qian ZM, Vaughn MG, Sauve S, Paul G, et al. Isomers of Perfluorooctanesulfonate (PFOS) in Cord Serum and Birth Outcomes in China: Guangzhou Birth Cohort Study. Environ Int (2017) 102:1-8. doi: 10.1016/j.envint.2017.03.006

45. Apelberg BJ, Witter FR, Herbstman JB, Calafat AM, Halden RU, Needham LL, et al. Cord Serum Concentrations of Perfluorooctane Sulfonate (PFOS) and Perfluorooctanoate (PFOA) in Relation to Weight and Size at Birth. Environ Health Perspect (2007) 115(11):1670-6. doi: 10.1289/ehp.10334

46. Chen MH, Ha EH, Wen TW, Su YN, Lien GW, Chen CY, et al. Perfluorinated Compounds in Umbilical Cord Blood and Adverse Birth Outcomes. PloS One (2012) 7(8):e42474. doi: 10.1371/journal.pone. 0042474

47. Shi Y, Yang L, Li J, Lai J, Wang Y, Zhao Y, et al. Occurrence of Perfluoroalkyl Substances in Cord Serum and Association With Growth Indicators in Newborns From Beijing. Chemosphere (2017) 169:396-402. doi: 10.1016/ j.chemosphere.2016.11.050

48. Chen MH, Ng S, Hsieh CJ, Lin CC, Hsieh WS, Chen PC. The Impact of Prenatal Perfluoroalkyl Substances Exposure on Neonatal and Child Growth. Sci Total Environ (2017) 607-608:669-75. doi: 10.1016/ j.scitotenv.2017.06.273

49. Stein CR, Savitz DA, Dougan M. Serum Levels of Perfluorooctanoic Acid and Perfluorooctane Sulfonate and Pregnancy Outcome. Am J Epidemiol (2009) 170(7):837-46. doi: 10.1093/aje/kwp212

50. Darrow LA, Stein CR, Steenland K. Serum Perfluorooctanoic Acid and Perfluorooctane Sulfonate Concentrations in Relation to Birth Outcomes in the Mid-Ohio Valley, 2005-2010. Environ Health Perspect (2013) 121 (10):1207-13. doi: 10.1289/ehp.1206372

51. Lee ES, Han S, Oh JE. Association Between Perfluorinated Compound Concentrations in Cord Serum and Birth Weight Using Multiple Regression Models. Reprod Toxicol (2016) 59:53-9. doi: 10.1016/ j.reprotox.2015.10.020 
52. Woods MM, Lanphear BP, Braun JM, McCandless LC. Gestational Exposure to Endocrine Disrupting Chemicals in Relation to Infant Birth Weight: A Bayesian Analysis of the HOME Study. Environ Health (2017) 16(1):115. doi: 10.1186/s12940-017-0332-3

53. Lee YJ, Kim MK, Bae J, Yang JH. Concentrations of Perfluoroalkyl Compounds in Maternal and Umbilical Cord Sera and Birth Outcomes in Korea. Chemosphere (2013) 90(5):1603-9. doi: 10.1016/j.chemosphere. 2012.08.035

54. Callan AC, Rotander A, Thompson K, Heyworth J, Mueller JF, Odland Jø, et al. Maternal Exposure to Perfluoroalkyl Acids Measured in Whole Blood and Birth Outcomes in Offspring. Sci Total Environ (2016) 569-570:1107-13. doi: 10.1016/j.scitotenv.2016.06.177

55. de Cock M, De Boer MR, Lamoree M, Legler J, Van De Bor M. Prenatal Exposure to Endocrine Disrupting Chemicals and Birth Weight-A Prospective Cohort Study. J Environ Sci Health A Tox Hazard Subst Environ Eng (2016) 51(2):178-85. doi: 10.1080/10934529.2015.1087753

56. Xu C, Yin S, Liu Y, Chen F, Zhong Z, Li F, et al. Prenatal Exposure to Chlorinated Polyfluoroalkyl Ether Sulfonic Acids and Perfluoroalkyl Acids: Potential Role of Maternal Determinants and Associations With Birth Outcomes. J Hazard Mater (2019) 380:120867. doi: 10.1016/ j.jhazmat.2019.120867

57. Marks KJ, Howards PP, Smarr MM, Flanders WD, Northstone K, Daniel $\mathrm{JH}$, et al. Prenatal Exposure to Mixtures of Persistent EndocrineDisrupting Chemicals and Birth Size in a Population-Based Cohort of British Girls. Epidemiology (2021) 32(4):573-82. doi: 10.1097/EDE.0000 000000001351

58. Hu JMY, Arbuckle TE, Janssen P, Lanphear BP, Zhuang LH, Braun JM, et al. Prenatal Exposure to Endocrine Disrupting Chemical Mixtures and Infant Birth Weight: A Bayesian Analysis Using Kernel Machine Regression. Environ Res (2021) 195:110749. doi: 10.1016/j.envres.2021.110749

59. Govarts E, Remy S, Bruckers L, Den Hond E, Sioen I, Nelen V, et al. Combined Effects of Prenatal Exposures to Environmental Chemicals on Birth Weight. Int J Environ Res Public Health (2016) 13(5):495. doi: 10.3390/ ijerph13050495

60. Rokoff LB, Rifas-Shiman SL, Coull BA, Cardenas A, Calafat AM, Ye X, et al. Cumulative Exposure to Environmental Pollutants During Early Pregnancy and Reduced Fetal Growth: The Project Viva Cohort. Environ Health (2018) 17(1):19. doi: 10.1186/s12940-018-0363-4

61. Kalloo G, Wellenius GA, McCandless L, Calafat AM, Sjodin A, Romano ME, et al. Exposures to Chemical Mixtures During Pregnancy and Neonatal Outcomes: The HOME Study. Environ Int (2020) 134:105219. doi: 10.1016/ j.envint.2019.105219

62. Buck Louis GM, Zhai S, Smarr MM, Grewal J, Zhang C, Grantz KL, et al. Endocrine Disruptors and Neonatal Anthropometry, NICHD Fetal Growth Studies - Singletons. Environ Int (2018) 119:515-26. doi: 10.1016/ j.envint.2018.07.024

63. Xiao C, Grandjean P, Valvi D, Nielsen F, Jensen TK, Weihe P, et al. Associations of Exposure to Perfluoroalkyl Substances With Thyroid Hormone Concentrations and Birth Size. J Clin Endocrinol Metab (2020) 105(3):735-45. doi: 10.1210/clinem/dgz147

64. Kwon EJ, Shin JS, Kim BM, Shah-Kulkarni S, Park H, Kho YL, et al. Prenatal Exposure to Perfluorinated Compounds Affects Birth Weight Through GSTM1 Polymorphism. J Occup Environ Med (2016) 58(6):e198-205. doi: $10.1097 /$ jom.0000000000000739

65. Kobayashi S, Azumi K, Goudarzi H, Araki A, Miyashita C, Kobayashi S, et al. Effects of Prenatal Perfluoroalkyl Acid Exposure on Cord Blood IGF2/H19 Methylation and Ponderal Index: The Hokkaido Study. J Exposure Sci Environ Epidemiol (2017) 27(3):251-9. doi: 10.1038/jes.2016.50

66. Valvi D, Oulhote $\mathrm{Y}$, Weihe $\mathrm{P}$, Dalgård $\mathrm{C}$, Bjerve KS, Steuerwald U, et al. Gestational Diabetes and Offspring Birth Size at Elevated Environmental Pollutant Exposures. Environ Int (2017) 107:205-15. doi: 10.1016/ j.envint.2017.07.016

67. Wang H, Du H, Yang J, Jiang H, Karmin O, Xu L, et al. PFOS, PFOA, Estrogen Homeostasis, and Birth Size in Chinese Infants. Chemosphere (2019) 221:349-55. doi: 10.1016/j.chemosphere.2019.01.061

68. Starling AP, Adgate JL, Hamman RF, Kechris K, Calafat AM, Ye X, et al. Perfluoroalkyl Substances During Pregnancy and Offspring Weight and Adiposity at Birth: Examining Mediation by Maternal Fasting Glucose in the
Healthy Start Study. Environ Health Perspect (2017) 125(6):067016. doi: 10.1289/EHP641

69. Negri E, Metruccio F, Guercio V, Tosti L, Benfenati E, Bonzi R, et al. Exposure to PFOA and PFOS and Fetal Growth: A Critical Merging of Toxicological and Epidemiological Data. Crit Rev Toxicol (2017) 47(6):482508. doi: 10.1080/10408444.2016.1271972

70. Steenland K, Barry V, Savitz D. Serum Perfluorooctanoic Acid and Birthweight: An Updated Meta-Analysis With Bias Analysis. Epidemiology (2018) 29(6):765-76. doi: 10.1097/EDE.0000000000000903

71. Lee YA, Kim JH, Jung HW, Lim YH, Bae S, Kho Y, et al. The Serum Concentrations of Perfluoroalkyl Compounds Were Inversely Associated With Growth Parameters in 2-Year Old Children. Sci Total Environ (2018) 628-629:226-32. doi: 10.1016/j.scitotenv.2018.02.050

72. Scinicariello F, Buser MC, Abadin HG, Attanasio R. Perfluoroalkyl Substances and Anthropomorphic Measures in Children (Ages 3-11 Years), NHANES 2013-2014. Environ Res (2020) 186:109518. doi: 10.1016/j.envres.2020.109518

73. Andersen CS, Fei C, Gamborg M, Nohr EA, Sørensen TIA, Olsen J. Prenatal Exposures to Perfluorinated Chemicals and Anthropometric Measures in Infancy. Am J Epidemiol (2010) 172(11):1230-7. doi: 10.1093/aje/kwq289

74. Shoaff J, Papandonatos GD, Calafat AM, Chen A, Lanphear BP, Ehrlich S, et al. Prenatal Exposure to Perfluoroalkyl Substances: Infant Birth Weight and Early Life Growth. Environ Epidemiol (2018) 2(2):e010. doi: 10.1097/ EE9.0000000000000010

75. Starling AP, Adgate JL, Hamman RF, Kechris K, Calafat AM, Dabelea D. Prenatal Exposure to Per- and Polyfluoroalkyl Substances and Infant Growth and Adiposity: The Healthy Start Study. Environ Int (2019) 131:104983. doi: 10.1016/j.envint.2019.104983

76. Gyllenhammar I, Diderholm B, Gustafsson J, Berger U, Ridefelt P, Benskin JP, et al. Perfluoroalkyl Acid Levels in First-Time Mothers in Relation to Offspring Weight Gain and Growth. Environ Int (2018) 111:191-9. doi: 10.1016/j.envint.2017.12.002

77. Cao W, Liu X, Liu X, Zhou Y, Zhang X, Tian H, et al. Perfluoroalkyl Substances in Umbilical Cord Serum and Gestational and Postnatal Growth in a Chinese Birth Cohort. Environ Int (2018) 116:197-205. doi: 10.1016/ j.envint.2018.04.015

78. de Cock M, de Boer MR, Lamoree M, Legler J, van de Bor M. First Year Growth in Relation to Prenatal Exposure to Endocrine Disruptors - a Dutch Prospective Cohort Study. Int J Environ Res Public Health (2014) 11 (7):7001-21. doi: 10.3390/ijerph110707001

79. Jensen RC, Andersen MS, Larsen PV, Glintborg D, Dalgard C, Timmermann CAG, et al. Prenatal Exposures to Perfluoroalkyl Acids and Associations With Markers of Adiposity and Plasma Lipids in Infancy: An Odense Child Cohort Study. Environ Health Perspect (2020) 128(7):77001. doi: 10.1289/ EHP5184

80. Mora AM, Oken E, Rifas-Shiman SL, Webster TF, Gillman MW, Calafat AM, et al. Prenatal Exposure to Perfluoroalkyl Substances and Adiposity in Early and Mid-Childhood. Environ Health Perspect (2017) 125(3):467-73. doi: 10.1289/EHP246

81. Andersen CS, Fei C, Gamborg M, Nohr EA, Sorensen TI, Olsen J. Prenatal Exposures to Perfluorinated Chemicals and Anthropometry at 7 Years of Age. Am J Epidemiol (2013) 178(6):921-7. doi: 10.1093/aje/kwt057

82. Manzano-Salgado CB, Casas M, Lopez-Espinosa MJ, Ballester F, Iniguez C, Martinez D, et al. Prenatal Exposure to Perfluoroalkyl Substances and Cardiometabolic Risk in Children From the Spanish INMA Birth Cohort Study. Environ Health Perspect (2017) 125(9):097018. doi: 10.1289/EHP1330

83. Braun JM, Eliot M, Papandonatos GD, Buckley JP, Cecil KM, Kalkwarf HJ, et al. Gestational Perfluoroalkyl Substance Exposure and Body Mass Index Trajectories Over the First 12 Years of Life. Int J Obes (Lond) (2021) 45 (1):25-35. doi: 10.1038/s41366-020-00717-x

84. Lauritzen HB, Larose TL, Oien T, Sandanger TM, Odland JO, van de Bor M, et al. Prenatal Exposure to Persistent Organic Pollutants and Child Overweight/Obesity at 5-Year Follow-Up: A Prospective Cohort Study. Environ Health (2018) 17(1):9. doi: 10.1186/s12940-017-0338-x

85. Braun JM, Chen A, Romano ME, Calafat AM, Webster GM, Yolton K, et al. Prenatal Perfluoroalkyl Substance Exposure and Child Adiposity at 8 Years of Age: The HOME Study. Obes (Silver Spring) (2016) 24(1):231-7. doi: $10.1002 /$ oby.21258 
86. Hoyer BB, Ramlau-Hansen CH, Vrijheid M, Valvi D, Pedersen HS, Zviezdai $\mathrm{V}$, et al. Anthropometry in 5- to 9-Year-Old Greenlandic and Ukrainian Children in Relation to Prenatal Exposure to Perfluorinated Alkyl Substances. Environ Health Perspect (2015) 123(8):841-6. doi: 10.1289/ ehp. 1408881

87. Hartman TJ, Calafat AM, Holmes AK, Marcus M, Northstone K, Flanders WD, et al. Prenatal Exposure to Perfluoroalkyl Substances and Body Fatness in Girls. Child Obes (2017) 13(3):222-30. doi: 10.1089/chi.2016.0126

88. Liu Y, Li N, Papandonatos GD, Calafat AM, Eaton CB, Kelsey KT, et al. Exposure to Per- And Polyfluoroalkyl Substances and Adiposity at Age 12 Years: Evaluating Periods of Susceptibility. Environ Sci Technol (2020) 54 (24):16039-49. doi: 10.1021/acs.est.0c06088

89. Martinsson M, Nielsen C, Bjork J, Rylander L, Malmqvist E, Lindh C, et al. Intrauterine Exposure to Perfluorinated Compounds and Overweight at Age 4: A Case-Control Study. PloS One (2020) 15(3):e0230137. doi: 10.1371/ journal.pone. 0230137

90. Halldorsson TI, Rytter D, Haug LS, Bech BH, Danielsen I, Becher G, et al. Prenatal Exposure to Perfluorooctanoate and Risk of Overweight at 20 Years of Age: A Prospective Cohort Study. Environ Health Perspect (2012) 120 (5):668-73. doi: 10.1289/ehp.1104034

91. Karlsen M, Grandjean P, Weihe P, Steuerwald U, Oulhote Y, Valvi D. EarlyLife Exposures to Persistent Organic Pollutants in Relation to Overweight in Preschool Children. Reprod Toxicol (2017) 68:145-53. doi: 10.1016/ j.reprotox.2016.08.002

92. Chen Q, Zhang X, Zhao Y, Lu W, Wu J, Zhao S, et al. Prenatal Exposure to Perfluorobutanesulfonic Acid and Childhood Adiposity: A Prospective Birth Cohort Study in Shanghai, China. Chemosphere (2019) 226:17-23. doi: 10.1016/j.chemosphere.2019.03.095

93. Geiger SD, Yao P, Vaughn MG, Qian Z. PFAS Exposure and Overweight/ Obesity Among Children in a Nationally Representative Sample. Chemosphere (2021) 268:128852. doi: 10.1016/j.chemosphere.2020.128852

94. Fassler CS, Pinney SE, Xie C, Biro FM, Pinney SM. Complex Relationships Between Perfluorooctanoate, Body Mass Index, Insulin Resistance and Serum Lipids in Young Girls. Environ Res (2019) 176:108558. doi: 10.1016/j.envres.2019.108558

95. Timmermann CAG, Rossing LI, Grøntved A, Ried-Larsen M, Dalgård C, Andersen LB, et al. Adiposity and Glycemic Control in Children Exposed to Perfluorinated Compounds. J Clin Endocrinol Metab (2014) 99(4):E608E14. doi: 10.1210/jc.2013-3460

96. Domazet SL, Grontved A, Timmermann AG, Nielsen F, Jensen TK. Longitudinal Associations of Exposure to Perfluoroalkylated Substances in Childhood and Adolescence and Indicators of Adiposity and Glucose Metabolism 6 and 12 Years Later: The European Youth Heart Study. Diabetes Care (2016) 39(10):1745-51. doi: 10.2337/dc16-0269

97. Averina M, Brox J, Huber S, Furberg AS. Exposure to Perfluoroalkyl Substances (PFAS) and Dyslipidemia, Hypertension and Obesity in Adolescents. The Fit Futures Study. Environ Res (2021) 195:110740. doi: 10.1016/j.envres.2021.110740

98. Mei Z, Grummer-Strawn LM, Thompson D, Dietz WH. Shifts in Percentiles of Growth During Early Childhood: Analysis of Longitudinal Data From the California Child Health and Development Study. Pediatrics (2004) 113(6): e617-27. doi: 10.1542/peds.113.6.e617

99. Greenspan LC, Lee MM. Endocrine Disrupters and Pubertal Timing. Curr Opin Endocrinol Diabetes Obes (2018) 25(1):49-54. doi: 10.1097/ MED.0000000000000377

100. Abreu AP, Kaiser UB. Pubertal Development and Regulation. Lancet Diabetes Endocrinol (2016) 4(3):254-64. doi: 10.1016/s2213-8587(15)00418-0

101. Lee JE, Jung HW, Lee YJ, Lee YA. Early-Life Exposure to EndocrineDisrupting Chemicals and Pubertal Development in Girls. Ann Pediatr Endocrinol Metab (2019) 24(2):78-91. doi: 10.6065/apem.2019.24.2.78

102. Ernst A, Brix N, Lauridsen LLB, Olsen J, Parner ET, Liew Z, et al. Exposure to Perfluoroalkyl Substances During Fetal Life and Pubertal Development in Boys and Girls From the Danish National Birth Cohort. Environ Health Perspect (2019) 127(1):17004. doi: 10.1289/EHP3567

103. Kristensen SL, Ramlau-Hansen CH, Ernst E, Olsen SF, Bonde JP, Vested A, et al. Long-Term Effects of Prenatal Exposure to Perfluoroalkyl Substances on Female Reproduction. Hum Reprod (2013) 28(12):3337-48. doi: 10.1093/ humrep/det382
104. Christensen KY, Maisonet M, Rubin C, Holmes A, Calafat AM, Kato K, et al. Exposure to Polyfluoroalkyl Chemicals During Pregnancy is Not Associated With Offspring Age at Menarche in a Contemporary British Cohort. Environ Int (2011) 37(1):129-35. doi: 10.1016/j.envint.2010.08.007

105. Marks KJ, Howards PP, Smarr MM, Flanders WD, Northstone K, Daniel JH, et al. Prenatal Exposure to Mixtures of Persistent Endocrine Disrupting Chemicals and Early Menarche in a Population-Based Cohort of British Girls. Environ Pollut (2021) 276:116705. doi: 10.1016/j.envpol.2021.116705

106. Maisonet M, Calafat AM, Marcus M, Jaakkola JJ, Lashen H. Prenatal Exposure to Perfluoroalkyl Acids and Serum Testosterone Concentrations at 15 Years of Age in Female ALSPAC Study Participants. Environ Health Perspect (2015) 123(12):1325-30. doi: 10.1289/ehp.1408847

107. Lopez-Espinosa MJ, Mondal D, Armstrong BG, Eskenazi B, Fletcher T. Perfluoroalkyl Substances, Sex Hormones, and Insulin-Like Growth Factor-1 at 6-9 Years of Age: A Cross-Sectional Analysis Within the C8 Health Project. Environ Health Perspect (2016) 124(8):1269-75. doi: 10.1289/ ehp.1509869

108. Lopez-Espinosa MJ, Fletcher T, Armstrong B, Genser B, Dhatariya K, Mondal D, et al. Association of Perfluorooctanoic Acid (PFOA) and Perfluorooctane Sulfonate (PFOS) With Age of Puberty Among Children Living Near a Chemical Plant. Environ Sci Technol (2011) 45(19):8160-6. doi: $10.1021 /$ es 1038694

109. Tsai MS, Lin CY, Lin CC, Chen MH, Hsu SH, Chien KL, et al. Association Between Perfluoroalkyl Substances and Reproductive Hormones in Adolescents and Young Adults. Int J Hyg Environ Health (2015) 218 (5):437-43. doi: 10.1016/j.ijheh.2015.03.008

110. Zhou Y, Hu LW, Qian ZM, Chang JJ, King C, Paul G, et al. Association of Perfluoroalkyl Substances Exposure With Reproductive Hormone Levels in Adolescents: By Sex Status. Environ Int (2016) 94:189-95. doi: 10.1016/ j.envint.2016.05.018

111. Lewis RC, Johns LE, Meeker JD. Serum Biomarkers of Exposure to Perfluoroalkyl Substances in Relation to Serum Testosterone and Measures of Thyroid Function Among Adults and Adolescents From NHANES 2011-2012. Int J Environ Res Public Health (2015) 12(6):6098-114. doi: 10.3390/ijerph120606098

112. Di Nisio A, Sabovic I, Valente U, Tescari S, Rocca MS, Guidolin D, et al. Endocrine Disruption of Androgenic Activity by Perfluoroalkyl Substances: Clinical and Experimental Evidence. J Clin Endocrinol Metab (2019) 104 (4):1259-71. doi: 10.1210/jc.2018-01855

113. Vanden Heuvel JP, Thompson JT, Frame SR, Gillies PJ. Differential Activation of Nuclear Receptors by Perfluorinated Fatty Acid Analogs and Natural Fatty Acids: A Comparison of Human, Mouse, and Rat Peroxisome Proliferator-Activated Receptor-Alpha, -Beta, and -Gamma, Liver X Receptor-Beta, and Retinoid X Receptor-Alpha. Toxicol Sci (2006) 92 (2):476-89. doi: 10.1093/toxsci/kfl014

114. Szilagyi JT, Avula V, Fry RC. Perfluoroalkyl Substances (PFAS) and Their Effects on the Placenta, Pregnancy, and Child Development: A Potential Mechanistic Role for Placental Peroxisome Proliferator-Activated Receptors (PPARs). Curr Environ Health Rep (2020) 7(3):222-30. doi: 10.1007/s40572020-00279-0

115. Abbott BD, Wolf CJ, Schmid JE, Das KP, Zehr RD, Helfant L, et al. Perfluorooctanoic Acid Induced Developmental Toxicity in the Mouse is Dependent on Expression of Peroxisome Proliferator Activated ReceptorAlpha. Toxicol Sci (2007) 98(2):571-81. doi: 10.1093/toxsci/kfm110

116. Khazaee M, Christie E, Cheng W, Michalsen M, Field J, Ng C. Perfluoroalkyl Acid Binding With Peroxisome Proliferator-Activated Receptors Alpha, Gamma, and Delta, and Fatty Acid Binding Proteins by Equilibrium Dialysis With a Comparison of Methods. Toxics (2021) 9(3):45. doi: $10.3390 /$ toxics 9030045

117. Ferre P. The Biology of Peroxisome Proliferator-Activated Receptors: Relationship With Lipid Metabolism and Insulin Sensitivity. Diabetes (2004) 53 Suppl 1:S43-50. doi: 10.2337/diabetes.53.2007.s43

118. Han L, Shen WJ, Bittner S, Kraemer FB, Azhar S. PPARs: Regulators of Metabolism and as Therapeutic Targets in Cardiovascular Disease. Part II: PPAR-Beta/Delta and PPAR-Gamma. Future Cardiol (2017) 13(3):279-96. doi: $10.2217 / \mathrm{fca}-2017-0019$

119. Lee JE, Choi K. Perfluoroalkyl Substances Exposure and Thyroid Hormones in Humans: Epidemiological Observations and Implications. Ann Pediatr Endocrinol Metab (2017) 22(1):6-14. doi: 10.6065/apem.2017.22.1.6 
120. Coperchini F, Croce L, Ricci G, Magri F, Rotondi M, Imbriani M, et al. Thyroid Disrupting Effects of Old and New Generation PFAS. Front Endocrinol (Lausanne) (2020) 11:612320. doi: 10.3389/fendo.2020.612320

121. Ye L, Guo J, Ge RS. Environmental Pollutants and Hydroxysteroid Dehydrogenases. Vitam Horm (2014) 94:349-90. doi: 10.1016/B978-0-12800095-3.00013-4

122. Li X, Ye L, Ge Y, Yuan K, Zhang Y, Liang Y, et al. In Utero Perfluorooctane Sulfonate Exposure Causes Low Body Weights of Fetal Rats: A Mechanism Study. Placenta (2016) 39:125-33. doi: 10.1016/j.placenta.2016.01.010

123. Zhang N, Wang WS, Li WJ, Liu C, Wang Y, Sun K. Reduction of Progesterone, Estradiol and hCG Secretion by Perfluorooctane Sulfonate via Induction of Apoptosis in Human Placental Syncytiotrophoblasts. Placenta (2015) 36(5):575-80. doi: 10.1016/j.placenta.2015.02.008

124. Ding N, Harlow SD, Randolph JF Jr, Loch-Caruso R, Park SK. Perfluoroalkyl and Polyfluoroalkyl Substances (PFAS) and Their Effects on the Ovary. Hum Reprod Update (2020) 26(5):724-52. doi: 10.1093/humupd/dmaa018

125. Kjeldsen LS, Bonefeld-Jorgensen EC. Perfluorinated Compounds Affect the Function of Sex Hormone Receptors. Environ Sci Pollut Res Int (2013) 20 (11):8031-44. doi: 10.1007/s11356-013-1753-3

126. Du G, Hu J, Huang Z, Yu M, Lu C, Wang X, et al. Neonatal and Juvenile Exposure to Perfluorooctanoate (PFOA) and Perfluorooctane Sulfonate (PFOS): Advance Puberty Onset and Kisspeptin System Disturbance in Female Rats. Ecotoxicol Environ Saf (2019) 167:412-21. doi: 10.1016/ j.ecoenv.2018.10.025

127. Braun JM. Early-Life Exposure to EDCs: Role in Childhood Obesity and Neurodevelopment. Nat Rev Endocrinol (2017) 13(3):161-73. doi: 10.1038/ nrendo.2016.186

128. Shalitin S, Kiess W. Putative Effects of Obesity on Linear Growth and Puberty. Horm Res Paediatr (2017) 88(1):101-10. doi: 10.1159/000455968

129. Zhao B, Chu Y, Hardy DO, Li XK, Ge RS. Inhibition of 3beta- and 17betaHydroxysteroid Dehydrogenase Activities in Rat Leydig Cells by Perfluorooctane Acid. J Steroid Biochem Mol Biol (2010) 118(1-2):13-7. doi: 10.1016/j.jsbmb.2009.09.010

130. Shi Z, Zhang H, Ding L, Feng Y, Xu M, Dai J. The Effect of Perfluorododecanonic Acid on Endocrine Status, Sex Hormones and Expression of Steroidogenic Genes in Pubertal Female Rats. Reprod Toxicol (2009) 27(3-4):352-9. doi: 10.1016/j.reprotox.2009.02.008

131. Street ME, Angelini S, Bernasconi S, Burgio E, Cassio A, Catellani C, et al. Current Knowledge on Endocrine Disrupting Chemicals (EDCs) From
Animal Biology to Humans, From Pregnancy to Adulthood: Highlights From a National Italian Meeting. Int J Mol Sci (2018) 19(6):1647. doi: 10.3390/ijms19061647

132. Ritscher A, Wang Z, Scheringer M, Boucher JM, Ahrens L, Berger U, et al. Zurich Statement on Future Actions on Per- and Polyfluoroalkyl Substances (PFASs). Environ Health Perspect (2018) 126(8):84502. doi: 10.1289/ EHP4158

133. Brendel S, Fetter E, Staude C, Vierke L, Biegel-Engler A. Short-Chain Perfluoroalkyl Acids: Environmental Concerns and a Regulatory Strategy Under REACH. Environ Sci Eur (2018) 30(1):9. doi: 10.1186/s12302-0180134-4

134. Ojo AF, Peng C, Ng JC. Assessing the Human Health Risks of Per- and Polyfluoroalkyl Substances: A Need for Greater Focus on Their Interactions as Mixtures. J Hazard Mater (2021) 407:124863. doi: 10.1016/ j.jhazmat.2020.124863

135. Braun JM, Gray K. Challenges to Studying the Health Effects of Early Life Environmental Chemical Exposures on Children's Health. PloS Biol (2017) 15(12):e2002800. doi: 10.1371/journal.pbio.2002800

136. Vandenberg LN, Colborn T, Hayes TB, Heindel JJ, Jacobs DRJr., Lee DH, et al. Hormones and Endocrine-Disrupting Chemicals: Low-Dose Effects and Nonmonotonic Dose Responses. Endocr Rev (2012) 33(3):378-455. doi: 10.1210/er.2011-1050

Conflict of Interest: The authors declare that the research was conducted in the absence of any commercial or financial relationships that could be construed as a potential conflict of interest.

Publisher's Note: All claims expressed in this article are solely those of the authors and do not necessarily represent those of their affiliated organizations, or those of the publisher, the editors and the reviewers. Any product that may be evaluated in this article, or claim that may be made by its manufacturer, is not guaranteed or endorsed by the publisher.

Copyright (c) 2021 Lee, Jung, Kim, Choi and Lee. This is an open-access article distributed under the terms of the Creative Commons Attribution License (CC BY). The use, distribution or reproduction in other forums is permitted, provided the original author(s) and the copyright owner(s) are credited and that the original publication in this journal is cited, in accordance with accepted academic practice. No use, distribution or reproduction is permitted which does not comply with these terms. 